\title{
Amortissement des houles dans le domaine de l'eau peu profonde
}

\author{
Wave decay in shallow water
}

\author{
PAR R. MICHE
}

DIRECTEUR DES ENTREPRISES DE grands travaUX hydRaUlgues

\begin{abstract}
Introduction.
Classification des houles selon leur profondeur relative.

I. Gradients de vitesse marginaux d'une houle dans un fuide visqueux: a) Calcul approché de la couche limile; b) Amortissement des houles en régime non turbulent; c) Introduction de nombres de Reynolds.

II. Examen de quelques résultats expérimentaux d'amortissement de houles : a) Examen visuel (ou enregistrement photographique); b) Détermination du coefficient spatial d'amortissement des houles; c) Détermination, en laboraloire, de courbes d'amortissement de houles; d) Recherches de M. Huon Li sur la stabilité des couches limites oscillantes. Commentaires des recherches précédentes; e) Examen de la zone marginale d'une houle de laboratoire.

III. Amortissement des houles en profondeur finie. Etat actuel de la question. Recherches d̀ entreprendre.

ANNEXE. Calcul du taux d'amortissement d'une houle dans un fluide visqueux de profondeur constante, lorsque la vitesse se comporte régllièrement près du fond.
\end{abstract}

\begin{abstract}
Introduction.
Classification of waves according to their relative depths.

I. Boundary velocity gradients of a wave in a viscous fluid : a) Approximate calculation of the boundary layer; b) Wave decay in a nonturbulent regimen; c) The introduction of Reynolds Numbers.

II. Examination of experimental results concerning wave decay; a) Visual examination (or photographic records); b) Determination of the wave decay spatial factor; c) The determination of wave decay curves in a laboratory; d) Mr. Huon Li's work on the stability of oscillating boundary layers. Comments on previous research; e) Examination of the boundary zone. of laboratory produced waves.

III. Calculation of wave decay in a fluid with finite depth. The present state of the problem. Research work still to be done.

Appendrx. Calculation of the rate of decay of a wave in a viscous fluid with constant depth, when the velocity behaves regularly neas the bottom.
\end{abstract}

\section{INTRODUCTION}

Dans une note parue dans LA HOUILLE BLANCHE de janvier-février 1956, M. C. Carry, complétant les recherches de diver's auteurs, dont M. BIESEL, a démontré l'existence, dans un fluide visqueux à l'état non turbulent de profondeur finie, constante, de houles en régime éfabli, dont le taux d'amortissement dans l'espace comporte un terme principal en $\vee^{1 / 2}$, v étant le coefficient de viscosité cinématique, très faible pour l'eau comme on sait. Ce calcul suppose des «petits mouvements » et, en accord avec les lois de l'écoulement laminaire dans les tubes capillaires (théorie de Poiseuille), une vitesse nulle au contact du fond.

M. BIESEL, commentant ce résultat, signale $q u ' i l$ divergerail de celui indiqué dans une remarque de l'ouvrage de lauteur sur les trains d'ondes océaniques $\left[9\right.$, p. 9.3 ${ }^{(1)}$, dans laquelle il est fait état d'un terme principal en $v$, nettement plus petit. Cetfe différence n'est pas surprenante, car ce dernier résultat, établi expres-

(1) Les indications entre crochets concernent les références bibliographiques. 
sément en vue de contrôler, dans ce cas parficulier, le degré d'approximation réalisable avec la méthode énergétique de calcul des amortissements, utilisée dans l'ouvrage précité. exige, en accord avec le principe de cette méthode $[9, p .51]$, que la condition de vitesse nulle sur le fond soit omise, les phénomènes marginaux, schématisés par une force représentative de frottement, devant être décomptés en sus. Si la présente note devait se borner à confirmer ce point, son intérêt serait fort limité. Toutefois, et ceci est plus important, le résultat en question, outre son aspect théorique, répond à une réalité expérimentale courante. Il résulte en effet de diverses constatations que, pour les conditions habituelles d'application (houles naturelles et de laboratoire), on aboutit d des solutions moins discutables en considérant une vitesse non nulle «sur le fond ». La signification à donner à ces derniers fermes et la justification de cette assertion entraînent à des développements qui débordent du cadre strictement théorique et amènent à examiner, avec quelque détail, la nafure complexe des mouvements de houle dans les fluides raturels, et leur état cinématique décelé par l'expérimentation.

A la lamière de ce qui précède, il apparaît ufile, d'une part, de fournir la preuve détaillée et, en tant que de besoin, préciser le sens du résultat indiqué trés sonmairement dans la remarque citée plus haut (1), d'autre part, de faire le point quant à l'état actuel de la question complexe évoquée ci-dessus, qui va constituer le sujet principal de cette note ( $\left.{ }^{2}\right)$.

Les faits essentiels semblent pouvoir se résumer comme suit:

La vifesse, au contact immédiat du fond, est très vraisemblablement nulle, mais si, dans ces conditions, on recherche, comme la fait après d'autres M. Carry, une solution de mouvements alternés répondant, quant à l'action des résistances passives, à une hypothèse Homogive dans toute la masse liquide, frontières y comprises (en l'espece, la loi du frottement visqueux de Newton-Navier), on se heurte, pour l'ensemble des houles réelles et la quasi-totalité de celles de laboratoire, à un obstacle sérieux qui rend très probablement illusoire un calcul de cette nature. Le gradient de vitesse est tellement élevé sur le fond qu'il semble difficile d'éviter l'apparition, dans son voisinage, d'une couche partiellement

(1) Cette démonstration est donnéc en Annexe.

(2) Le but poursuivi est l'examen détaillé des phénomènes et non l'élaboration de formules théoriques qui apparaîtraient encore prématurées. Au point de vue strictement utilitaire du calcul des amortissements effectifs de houles, les formules de caractère synthétique, basées notamment sur la méthode énergétique et n'exigreant pas la connaissance complète des mouvements peuvent suffire, pour l'instant du moins, car leur accord avec l'expérience paraît acceptable [ef., par exemple, 9, $2^{\circ}$ partic]. ou totalement turbulente. Or, celle-ci devrait être exclue par définition, sinon, le problème se présente de façon fort différente. Cette présomption théorique (et ressortant au surplus de certains essais) parait entièrement confirmée par la forme expérimentale des courbes d'amortissement, qui n'a pas l'allure escomptée.

Ces constafations incitent à considérer le probleme des houles courantes de laboratoire ou naturelles d'une facon assez analogue à celle envisagée par Prandtl et ses continuateurs pour les écoulements furbulents, c'est-à-dire en admettant, d'une part, un film laminaire très mince attenant a la paroi, de l'autre un mouvement principal (enticrement turbulent dans le cas de Prandtl et comportant, dans le cas présent, une couche de caractère non laminaire près du fond, dont l'épaisseur croîtra avec les vitesses, c'esta-dire avec les amplitudes en jeu, le complément pouvant être, éventuellement, non turbulent), dont la vitesse dite « à la paroi » est déjà do l'ordre de grandeur de celles se produisant dans la masse liquide. Ceci conduit à l'analyse distincte de la parfie du mouvement principal située à l'intérieur de la masse liquide et des mouvements marginaux laminaire et turbulent. Pour le mouvement principal, la condition de vitesse nulle «sur le fond » n'a dès lors plus de raison d'être.

Les quotes-parts d'énergie dissipée sont, en grande majorite, concentrées dans les zones turbulentes (même relativement minces) et sont déterminantes pour la forme de la courbe d'amortissement. Ceci restera vrai pour des houles assez peu accusces pour que la presque totalité de la masse liquide soit à l'état non turbulent. Dans ce cas, la quote-part d'amorfissement due au mouvement visqueux interne (proportionnclle $\dot{a}$ v comme on l'a signalé ci-dessus), conservera une valeur subordonnée à celle due à la partie non laminaire de la courbe marginale. Si, enfin, la houle était assez faible pour correspondre $\dot{a}$ un mouvement enfierement non turbulent (solution de M. Carry), la quote-part de dissipation interne (proportionnelle $\dot{a}$ v) serait aussi subordonnée à celle due à la couche marginale laminaire (proportionnelle $\dot{a} v^{1 / 2}$ ), mais, dans ce cas, la forme de la courbe d'amortissement serait différente. Il semble, si l'on en juge d'après les essais analysés, que de telles honles devraient être d'amplitude quasi micrométrique, car même pour les houles les plus faibles de laboratoire expérimentées. cefte allure de la courbe d'amortissement n'a pas été observée.

Des essais récents de M. Huon II [5] laisseraient toutefois supposer un domaine d'application des régimes enfièrement non turbulents plus étendu qu'il ne résulterait des indications cidessus. Ces essais ne concernent pas strictement le problème des houles et certains contrôles évo- 
qués plus loin semblent montrer que pour ce cas, au contraire, les divergences précédentes, $\dot{a}$ supposer qu'elles subsistent encore, sont fortement atténuées.

Au surplus, la question ne peut être considérée comme entièrement élucidée, et l'un des buts de cette note, et non le moindre, serait de susciter de nouvelles recherches expérimentales. Le problème comporte, en effet, une série d'incidences importantes d'ordre pratique, en liaison, notamment, avec la stabilifé ou l'instabilité des fonds mobiles.

\section{I. - GRADIENTS DE VITESSE MARGINAUX D'UNE HOULE DANS UN FLUIDE VISQUEUX}

L'analy'se des houles naturelles montre qu'elles comportent toujours un spectre de périodes. Toutefois, pour les houles dites régulières, ce spectre est étroit, ce qui conduit, à titre approché, à la notion d'une houle monopériodique, telle qu'on s'efforce, en général, de la réaliser en laboratoire. On admettra ci-après des ondulations assez régulières pour que ce schéma soit acceptable. De plus, tous les mouvements envisagés seront bi-dimensionnels.

On sait qu'on peut classer les houles selon leur profondeur relative (rapport de la profondeur de l'eau $\mathrm{H}$ à la longueur d'onde $2 \mathrm{~L}$ ). Lorsque $\mathrm{H}: 2 \mathrm{~L}>1 / 2$, l'eau est dite profonde, et les mouvements près $\mathrm{du}$ fond sont presque insensibles. Lorsque $\mathrm{H}: 2 \mathrm{~L}<1 / 8$ environ, l'eau est dite peu profonde et les mouvements alternés près du fond sont à peine atténués par rapport à ceux de surface. On dit aussi que les ondes sont longues. Les deux domaines précédents et le domaine intermédiaire (eau assez profonde) doivent être envisagés pour les applications. Toutefois, le problème qui va nous occuper étant essentiellement l'étude des mouvements marginaux, on fera ci-après, et en général, abstraction du domaine de l'eau profonde et souvent même de celui de l'eau assez profonde. Quoique pour les houles se propageant dans un fluide naturel, les mouvements près du fond restent très notables dans la zone peu profonde, on admet ordinairement, en se basant sur les résultats obtenus lors de l'écoulement dans des tubes capillaires, que la vitesse au contact immédiat du fond est nulle. Le fluide adhérerait à la paroi et ceci conduit à envisager l'existence d'une couche marginale ou limite à fort gradient de vitesse.

\section{a) Calcul approché de la couche limite :}

Divers auteurs (cf. notamment [9, p. 93]), en partant d'une vitesse nulle sur le fond, en supposant, de plus, une profondeur constante, un fond lisse, et un régime non turbulent, ont été conduits à des taux d'amortissement de houle, soit dans le temps, soit dans l'espace, proportionnelle à $\nu^{1 / 2}(\nu=$ coefficient de viscosité cinématique du fluide, très faible pour de l'eau, soit environ $10^{-2} \mathrm{~cm}^{2} / \mathrm{s}$ à $\left.20^{\circ}\right)$. Le calcul plus rigoureux de M. Carry [3] donne le même résultat. Un moyen plus rapide de l'obtenir, permettant de définir, au préalable, la couche limite (cf. sur ce dernier point $[6],[10],\left[11_{1}^{1}\right)$, est le suivant :

Soit un mouvement horizontal et laminaire de vitesse $u$. Si l'on néglige le potentiel de gravité et les termes d'inertie de forme quadratique, la seule équation de Navier à satisfaire est :

$$
\frac{\partial u}{\partial t}=\nu\left(\frac{\partial^{2} u}{\partial x^{2}}+\frac{\partial^{2} u}{\partial z^{2}}\right)+\mathrm{X}
$$

Considérons, par exemple, un écoulement oscilIatoire :

$$
u=u_{m} \sin b t
$$

$u_{m}=$ vitesse maximum, $b=\frac{2 \pi}{2 \mathrm{~T}}, 2 \mathrm{~T}=$ période

Pour qu'un tel mouvement satisfasse à l'équation (1) et se raccorde à une vitesse nulle sur le fond, il doit être complété sous la forme :

$$
u=u_{m}\left[\sin b t-e^{-z / z_{0}} \sin \left(b t-z / z_{0}\right)\right],
$$

$z$ représentant l'ordonnée au-dessus du fond. La force $X$ n'est pas nulle, mais oscillante, et sert à entretenir le mouvement.

Dès que $z$ est grand vis-à-vis de la constante :

$$
z_{0}=\sqrt{\frac{2 v}{b}}=0,56 \sqrt{2 \mathrm{~T} v},
$$

(3) se ramène à (2). Par contre, $u$ est rapidement décroissant pour $z<z_{0}$, ce qui localise la couche limife.

La forme plus exacte de cette dernière ressort de la figure 2, représentant les valeurs maxima de $u$ en fonction de $z$, qu'on obtiendrait par la solution de M. Carry avec les données numériques indiquées.

Pour les besoins courants, la couche limite peut être caractérisée par l'épaisseur $z_{0}$. Celle-ci est telle que, si le gradient de vitesse $(\partial u / \partial z)$ était égal, dans toute la couche, à sa valeur $(\partial u / \partial z)_{0}$ sur le fond, au temps $t$, tel que $\sin b t=1$, 
la vitesse maximum à distance $z_{0}$ du fond serait égale à $u_{n_{0}}\left({ }^{1}\right)$.

Un tel mouvement peut se schématiser (dans le cas des houles longues), comme indiqué sur la figure 1, en discriminant la couche limite et

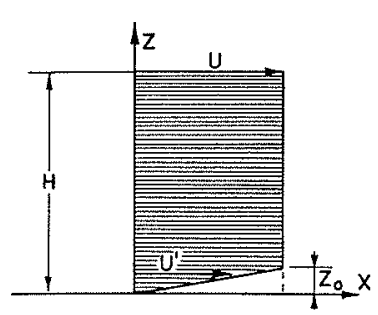

FrG. 1

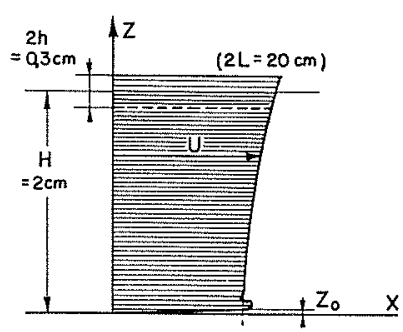

Fig. 2 un mouvement ondulatoire principal répondant aux lois des fluides parfaits et valable dans toute la masse liquide, couche marginale exclue.

Pour de l'eau, $z_{0}$ est très faible $(0,56 \mathrm{~mm}$ par exemple pour une houle de $1 \mathrm{~s}$ de période) et le gradient de vitesse correspondant:

$$
\left(\frac{\partial u}{\partial z}\right)_{0}=\frac{u_{m}}{z_{0}}
$$

est ordinairement très grand.

Le calcul approché précédent, qui néglige au surplus le déphasage de l'onde en fonction de l'abscisse $x$, s'applique le mieux aux ondes longues, avec amplitudes $2 h$ assez faibles pour qu'on puisse négliger les composantes verticales de la vitesse.

Toutefois, il reste utilisable dans tous les cas.

Soit, par exemple :

$u=u_{m} \sin (b t-a x), \quad a=\frac{2 \pi}{2 \mathrm{~L}}$,

$$
2 \mathrm{~L}=\text { longueur d'onde, }
$$

l'équation donnant les vitesses près du fond pour le mouvement principal. On aura les relations suivantes :

$$
\text { Célérité : } \begin{aligned}
\mathrm{V} & =\frac{b}{a}=\frac{2 \mathrm{~L}}{2 \mathrm{~T}} \\
& =\sqrt{\frac{g}{a} \text { th } a \mathrm{H}}, \quad[\sqrt{g \mathrm{H}}]
\end{aligned}
$$

Vitesse $u_{m}$ près du fond en fonction de l'amplitude $2 h$ :

$$
u_{m}=\frac{b h}{\operatorname{sh} a \mathrm{H}}, \quad\left[\frac{V h}{\mathrm{H}}\right]
$$

(1) Dans [11], M. Valembors adopte une épaisseur $d_{0}=z_{0}: \sqrt{2}$ correspondant au maximum maximorum $d u$ gradient marginal obtenu pour $\sin b t=\sqrt[4]{2} / 2$. C'est essentiellement une question de convention. Pour les calculs approchés que nous avons en vue, $z_{0}$ fournit une meilleure approximation et se relie plus directement au calcul rigoureux de M. Garry.
Excursion totale des particules près du fond :

$$
2 x=\frac{2 h}{\operatorname{sh} a \mathrm{H}}=\frac{2 u_{m}}{b}, \quad\left[\frac{2 h}{a \mathrm{H}}\right]
$$

Les expressions de droite entre crochets se rapportent au cas des houles longues ( $a \mathrm{H}$ petit), pour lesquelles, en outre, la vitesse maximum $u_{m}$ est sensiblement indépendante de $z$. Pour les houles non longues, par contre, $u_{m}$ croît constamment avec $z$ et atteint son maximum en surface, A titre d'exemple (fig. 2), admettons une houle des dimensions les plus faibles produites en laboratoire : $2 \mathrm{~L}=20 \mathrm{~cm}$., $\mathrm{H}=2 \mathrm{~cm}, 2 h=0,3 \mathrm{~cm}$ qu'on peut, avec une approximation encore acceptable, considérer comme longue. On aura :

$$
\begin{gathered}
\mathrm{V}=44 \mathrm{~cm} / \mathrm{s}, \quad 2 \mathrm{~T}=0,46 \mathrm{~s}, \quad u_{m}=3,3 \mathrm{~cm} / \mathrm{s} \\
2 r=0,48 \mathrm{~cm}, \quad z_{0}=0,38 \mathrm{~mm}
\end{gathered}
$$

enfin :

$$
\left(\frac{\partial u}{\partial z}\right)_{0}=87
$$

\section{b) Amortissement des houles en régime non turbulent :}

Lorsqu'une houle se propage, même en profondeur constante, son amplitude varie, en général, au cours du temps et dans l'espace, par suite de l'action des résistances passives. Par contre, sa longueur d'onde reste pratiquement inchangée. Ơn peut évaluer ces variations d'amplitude, par la méthode dite énergétique, en utilisant l'équation du bilan d'énergie $[9, p .68]$ :

$$
\frac{\partial \mathcal{E}}{\partial x}+\frac{\partial \mathrm{E}}{\partial t}+\mathrm{Q}=0 \text {. }
$$

$\mathcal{G}=$ flux moyen d'énergie $=\mathrm{EG} ; \mathrm{E}=$ énergie unitaire totale $=\rho g\left(h^{2} / 2\right), \quad \rho=$ densité, $\mathrm{G}=$ vitesse de groupe $=q \mathrm{~V}$, où :

$$
q=\frac{1}{2}\left[1+\frac{2 a \mathrm{H}}{\operatorname{sh} 2 a \mathrm{H}}\right]
$$

$Q=$ énergie unitaire moyenne dissipée par les résistances passives et calculée ordinairement à partir des données du mouvement de base.

Pour un régime établi (1), le seul que nous envisageons, $\partial \mathrm{E} / \partial t=0$, et l'équation (11) peut s'écrire :

$$
\frac{\Delta h}{h}=\Delta \mathfrak{f} h=-\frac{\mathrm{Q}}{\rho g \mathrm{G} h^{2}} \Delta x,
$$

(1) C'est le cas le plus utile pour les essais de laboratoire et, d'ailleurs, celui envisagé par M. Canny. Pour un régime établi, les amplitudes sont, en général, fonctions de l'abscisse mais non du temps. 
le symbole $\Delta$ se rapportant à des différences réputées petites (en théorie à des différentielles). Dans le cas des houles longues, on pourra poser $\mathrm{G}=\mathrm{V}$.

Si le fluide est en régime non turbulent, $Q$ est donné par la valeur moyenne de l'intégrale de la fonction de dissipation :

$2 \Psi_{d}=\hat{p}\left\{2\left(\frac{\partial u}{\partial x}\right)^{2}+2\left(\frac{\partial w}{\partial z}\right)^{2}+\left(\frac{\partial u}{\partial z}+\frac{\partial w}{\partial x}\right)^{2}\right\}$

étendue à une tranche de longueur unité et de profondeur $\mathrm{H}$.

On trouve ainsi, pour le mouvement principal, en négligeant $z_{0}$ par rapport à $\mathrm{H}$ :

$$
\mathrm{Q}_{1, \nu}=2 \rho g \vee h^{2} a^{2} \text {. }
$$

Il faut tenir compte maintenant de la couche limite $0 \leqslant z \leqslant z_{0}$, pour laquelle :

et :

$$
u^{\prime}=u \frac{z}{z_{0}}
$$

$$
\frac{\partial u^{\prime}}{\partial z}=\frac{u}{z_{0}} .
$$

Ce gradient a une valeur beaucoup plus grande que celle de $(\partial u / \partial x)$ qu'on peut négliger. Il viendra, dans ces conditions, d'après (14) :

$$
2 \Psi_{a}=\rho \vee\left(\frac{\partial u^{\prime}}{\partial z}\right)^{2}=\rho \vee \frac{u^{2}}{z_{0}^{2}}
$$

d'où, en intégrant pour une tranche d'épaisseur $z_{0}$ et de longueur unité, puis en calculant la valeur moyenne correspondante :

$$
\mathrm{Q}_{2, \nu}=\frac{\rho \nu}{2} \frac{u_{m}{ }^{2}}{z_{0}}=\rho \frac{v^{1 / 2}}{2 \sqrt{2}} \frac{b^{5 / 2} h^{2}}{\operatorname{sh}^{2} a \mathrm{H}} .
$$

$Q_{2, \nu}$, proportionnel à $\nu^{1 / 2}$, sera en général nettement plus grand que $Q_{1, \nu}$, proportionnel à \%

En résumé, dans le cas des mouvements non turbulents, la dissipation d'énergie:

$$
\mathrm{Q}=\mathrm{Q}_{1, \nu}+\mathrm{Q}_{2, \nu}
$$

se réalise, à titre accessoire, à l'intérieur de la masse liquide et, en majorité, dans la couche marginale ${ }^{1}$ ).

L'une et l'autre des deux quotes-parts précédentes sont proportionnelles à $h^{2}$ et, par conséquent, selon la formule (13), le taux d'amortissement de l'amplitude par unité de longueur de cheminement, soit $\Delta \mathscr{S}_{\mathrm{s}} \mathrm{\Delta} / \Delta x$, est indépendant de l'amplitude. L'intégration de l'équation (13) conduit, dans ce cas, à une courbe de décroissance

(1) On néglige ici les dissipations annexes d'énergie, celle due au freinage de l'air, par exemple. des amplitudes de forme exponentielle par rapport à l'abscisse $x\left(^{(2)}\right.$.

\section{c) Introduction de nombres de Reynolds :}

Pour que les calculs précédents correspondent à la réalité, il est nécessaire que la loi du frottement visqueux de Newton :

$$
\tau=\rho \nu \frac{\partial u}{\partial z}
$$

( $\tau$ effort tangentiel en direction $x, \partial u / \partial z$ gradient de vitesse en direction perpendiculaire), servant de base au calcul de la fonction de dissipation $2 \Psi_{d}$ soit valable dans tout le profil liquide. Autrement dit, il ne doit pas exister de zones turbulentes, même localisées, car, pour ces dernières, le coefficient $\rho \vee$ de la formule (19) cesse d'être constant et peut atteindre des valeurs considérables. Une région non laminaire, même de faible étendue, pourra donc modifier de façon très importante les lois d'amortissement.

Les critères de turbulence, partielle ou complète, sont en liaison avec certains paramètres non dimensionnels. Dans les cas courants, on se contente, ordinairement, d'un seul paramètre dit nombre de Reynolds, représentant le produit d'une vitesse caractéristique par une dimension caractéristique de l'écoulement, le tout divisé par le coefficient de viscosité cinématique y. Cette définition, de nature conventionnelle, doit être précisée dans chaque cas.

Le plus simple d'entre eux, qui va nous permettre de tirer une première conclusion d'ordre général sur le comportement des mouvements ondulatoires, est celui d'un écoulement laminaire et permanent d'un fluide visqueux d'épaisseur $\mathbf{H}$, s'effectuant sur un plan (fig. 3). La

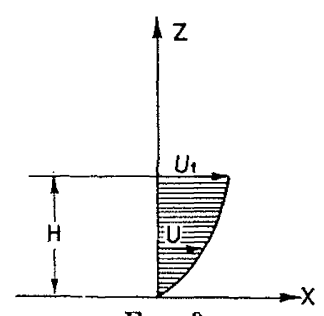

(2) Si l'on compare les résultats approchés précédents à ceux obtenus par M. Carro, qui doivent être considérés comme rigoureux, à l'approximation des petits mouvements, on aboutit aux constatations suivantes quant aux lois d'amortissement des amplitudes :

a) le terme d'amortissement principal dépendant de $Q_{2, \nu}$ et proportionnel $\dot{a} v^{1 / 2}$ est identique dans les deux cas, ce qui constitue une justification d'ordre général du mode d’approximation adopté, bilan énergétique y compris, et confirme l'intérêt du choix de $z_{0}$ comme épaisseur de la couche limite;

b) le terme suivant, proportionnel à $v$ et ressortant de $Q_{1, \nu}$ est de forme différente, mais sa valess numérique est proche de celle donnée par la théorie rigoureuse, sauf si l'eau est relativement très peu profonde. 
répartition des vitesses répondant aux équations de Navier est alors parabolique.

$$
\left.u=u_{1} 1-\left(\frac{\mathrm{H}-z}{\mathrm{H}}\right)^{2}\right] .
$$

Le gradient de vitesse, nul en surface $(z=\mathrm{H})$, est maximum sur le fond $(z=0)$ et vaut :

$$
\left(\frac{\partial u}{\partial z}\right)_{0}=\frac{2 u_{1}}{\mathrm{H}}
$$

On pourra adopter, comme éléments caractéristiques de l'écoulement, la vitesse moyenne $\vec{u}=2 / 3 u_{1}$, et le rayon hydraulique $R$, identique à $\mathrm{H}$ dans le cas présent. Le nombre de Reynolds sera, dans ces conditions, égal à :

$$
\mathcal{R}=\frac{\bar{u} \mathrm{R}}{\nu}=\frac{2}{3} u_{1} \frac{\mathrm{H}}{\nu}=\frac{\mathrm{H}^{2}}{3 \nu}\left(\frac{\partial u}{\partial z}\right)_{0}
$$

Un tel écoulement a tendance à devenir turbulent dès que ${ }^{(1)}$ :

$$
\mathfrak{R}>\mathfrak{R}_{c}=\frac{2200}{4}=550 \mathrm{env} .
$$

Par conséquent, le gradient de vitesse maximum compatible avec un mouvement inconditionnellement laminaire sera donné par :

$$
\left(\frac{\partial u}{\partial z}\right)_{0} \leqslant \frac{3 \nu}{\mathrm{H}^{2}} \mathfrak{R}_{c}=\frac{16,5}{\mathrm{H}^{2}}
$$

II vaudra 4,1 par exemple, si $\mathrm{H}=2 \mathrm{~cm}$, et sera, dans ce cas : $87: 4,1=21$ fois plus petit que le gradient marginal (10) obtenu pour le cas de houle envisagé ci-dessus pour la même profondeur H. Encore s'agit-il d'ondulations choisies parmi les plus faibles possibles. Pour les houles courantes utilisées en laboratoire et pour les houles réelles, le rapport précédent dépassera ordinairement 100 et pourra atteindre plusieurs millions. Si donc les alternances de courant sont assez lentes pour pouvoir être négligées (onde marée par exemple), il n'y a aucun doute possible. De tels mouvements de houle ne peuvent correspondre $\dot{a}$ des mouvements non turbulents dans leur ensemble et, par conséquent, un calcul d'amortissement, selon la loi (13) est illusoire. La conclusion n'est plus évidente et la question va se compliquer si les alternances de courant sont plus rapides, ou si les excursions des particules, près du fond, sont très petites.

Pour s'en rendre compte, il faut analyser plus en détail le comportement des couches limites

(1) Pour une conduite circulaire, de diamètre $d=4 \mathrm{R}$, on rapporte, ordinairement, le nombre de Reynolds au diamètre. L'expérience indique, dans ce cas :

$$
\mathfrak{R}_{e}=2.100 \text { à } 2.200 \mathrm{env} \text {. }
$$

pour des mouvements localement variables ou des écoulements non permanents. Avant d'examiner l'état actuel de la question au point de vue expérimental, il est indiqué de rechercher quels paramètres non dimensionnels analogues à des nombres de Reynolds, sont a priori susceptibles de correspondre à un mouvement de houle de la nature envisagée.

Par analogie avec l'écoulement permanent, on peut d'abord considérer un nombre de Reynolds global, qu'on dénommera $\mathfrak{K}_{1}$, basé sur les éléments caractéristiques suivants : pour les longueurs, le rayon hydraulique $\mathrm{R}=\mathrm{H}$; pour les vitesses, la composante horizontale maximum moyenne $\bar{u}_{m}$ au moment du passage des particules au droit de leur position de repos, soit $\mathrm{L} / \mathrm{T} . h / \mathrm{H}[9, \mathrm{p} .72]$, d'où :

$$
\mathfrak{o}_{1}=\frac{\bar{u}_{m} \mathrm{H}}{\nu}=\frac{\mathrm{V} h}{\nu}
$$

En envisageant plus spécialement la couche limite, on peut ensuite définir un nombre de Reynolds marginal :

$$
\boldsymbol{\alpha}_{2}=\frac{u_{m} \times z_{0}}{\nu}
$$

$u_{m}$ étant la vitesse maximum près du fond, $z_{0}$ l'épaisseur de la couche limite. Sauf dans le domaine de l'eau profonde, $u_{n}$ varie assez peu avec la profondeur (et même aucunement si les ondes sont longues). $\alpha_{2}$ apparait donc plus ou moins indépendant de la profondeur $\mathrm{H}$; par contre, il est fonction de $b$, c'est-à-dire de la périodé $d u$ monvement d'après la valeur (4) de $z_{0}$.

On serait tenté, à première vue, d'introduire un troisième paramètre $\left(n_{m} \times 2 r\right) / \nu$ traduisant l'influence de l'excursion des particules près du fond. En réalité, ce n'est pas une entité distincte, car il ressort des relations (8) et (9) que ce paramètre représente le carré du précédent.

Par contre, il existe un autre facteur à prendre en compte : la rugosité du fond. Une paroi lísse est une pure abstraction, en tout cas pour les houles expérimentales ou réelles, d'où la nécessité de l'introduction d'un troisième paramètre non dimensionnel. On adopte souvent :

$$
\mathcal{R}_{3}=\frac{u_{m} \times \varepsilon}{\nu}
$$

$\varepsilon$ représentant une dimension caractéristique de la rugosité (par exemple la hauteur moyenne des aspérités ou le diamètre moyen des grains d'un fond sableux). La valeur critique de $\boldsymbol{R}_{3}$ susceptible de déclencher une turbulence varie d'ailleurs, assez nettement, avec la nature particulière du fond, même pour un $\varepsilon$ donné; autrement dit, l'expression (27) traduit de façon schématique une réalité plus complexe. En outre, et en toute rigueur, un quatrième facteur de tur- 
bulence devrait également jouer : le temps d'adaptation nécessaire à un régime ondulatoire donné pour que l'état cinématique définitif du mouvement soit réalisé. Dans le cas d'un régime sensiblement établi, pour lequel les mesures concernent, en général, des moyeunes relatives à un assez grand nombre d'ondes successives, il semble que l'on puisse faire abstraction de ce facteur. Il est en effet improbable que ce temps d'adaptation soit d'une durée telle que les mesures effectuées puissent en être affectées.

En résumé, une houle monopériodique progressant en profondeur finie constante paraît dépendre de trois paramètres non dimensionnels en ce qui concerne la fixation des caractères cinématiques du mouvement produit. Si le fond est réputé lisse, deux paramètres seraient même suffisants, alors que la houle elle-même dépend, dans ce cas, de trois paramètres indépendants : l'amplitude $2 h$, la profondeur $\mathrm{H}$ et la fréquence $b / 2 \pi$ (le nombre d'ondes $a / 2 \pi$ est fonction de $b$ et $H$ d'après la relation (7) dérivant de la condition de pression constante en surface). I'introduction de « nombres de Reynolds $\gg$ représente donc une simplification importante, qu'on peut également constater dans le cas de l'écoulement permanent, sur fond lisse par exemple (il existe alors un seul nombre de Reynolds (22) à opposer aux deux paramctres indépendants $u_{1}$ et $\mathrm{H}$ ).

Il n'en subsiste pas moins que l'étude détaillée de mouvements alternés pouvant comporter des zones turbulentes représente un problème complexe, non entièrement résolu d'ailleurs, et qu'il faudra tôt ou tard élucider. En effet, son intérêt n'est pas seulement théorique, car une série de faits importants en dépendent : lois déterminant l'érosion de fonds affouillables, amortissement des houles sur de longues distances et même à l'intérieur des ports, etc...

\section{II. - EXAMEN DE QUELQUES RESULTATS EXPÉRIMENTAUX D'AMORTISSEMENT DE HOULES}

On dispose de deux modes opératoires principaux pour déterminer ou, du moins, estimer l'état cinématique des mouvements de houle et notamment la turbulence.

\section{a) Examen visuel (ou enregistrement photo- graphique) des mouvements :}

Ordinairement, on se sert, pour déceler la turbulence, des traînées colorées produites par la dissolution de cristaux de permanganate de potasse. Sans méconnaître les mérites de tels essais, surtout à titre d'information générale, ils sont assez subjectifs. Leur nature qualifative ne permet pas la détermination du facteur essentiel : l'importance des dissipations d'énergie. Des mesures par thermo-couples électriques permettant d'apprécier, en une série de points, les quotes-parts d'énergie cinétique transformée en énergie calorifique seraient bien préférables, mais elles seraient très laborieuses et, semblet-il, de réalisation assez difficile.

\section{b) Détermination du coefficient spatial d'amortissement des houles :}

On renonce, dans ce cas, à l'examen détaillé du mouvement et se borne à apprécier l'effét résultant des dissipations d'énergie. Ce procédé, moins satisfaisant pour l'analyste et dont la réalisation n'est pas sans difficultés, a toutefois le gros avantage d'être d'ordre quantitatif. II n'est pas, non plus, à l'abri de toute critique d'ordre théorique; néanmoins, en l'état actuel de l'expérimentation, c'est sans doute le mode d'investigation le moins imparfait.

L'application du procédé conduit à utiliser l'équation (11) du bilan d'énergie, en schématisant l'action des résistances passives par une force de frottement qui, par exemple, sera supposée varier comme la puissance $n^{\text {irme }}$ d'une vitesse caractéristique, $n$ étant, a priori, indéterminé. Dans ce cas, l'énergie dissipée $Q$ sera proportionnelle à $h^{n+1}$ et, en intégrant l'équation (11), on obtiendra la courbe d'amortissement dans l'espace des amplitudes $[9$, p. 86 et suiv.].

Cette courbe sera exponentielle si l'exposant $n$ est́ égal à l'unité (force de frottement proportionnelle aux vitesses); en effet, $Q$ est alors proportionnel à $h^{2}$ et, en accord avec la formule (13), le taux unitaire d'amortissement de l'amplitude sera indépendant de cette dernière, ce qui conduit à une relation exponentielle. Si, par contre, $n$ est diflérent de $u n$, la courbe d'amortissement sera d'un type hyperbolique général en fonction de l'abscisse $x$. Notamment, si $n=2$, cas correspondant à une turbulence bien caractérisée (force de frottement proportionnelle au carré des vitesses), Q est proportionnel à $h^{3}$, et la conrbe d'amortissement représente une hyperbole équilatère. Le procédé est d'ailleurs très souple, en ce sens qu'il permet d'ajouter les quotes-parts d'énergie dissipées relatives, par exemple, aux différentes régions de la tranche liquide 
et dont la somme constituera la valeur $Q$. Ainsi, dans le cas laminaire avec couche limite traité plus haut, $Q$ sera la somme de deux dissipations d'énergie, l'une $Q_{1, \nu}$ localisée dans la masse liquide, l'autre $Q_{2, v}$ dans la couche limite (formule (18). A chacune de ces quotes-parts correspondra un amortissement unitaire dont la somme représentera l'amortissement unitaire résultant. On peut aussi supposer une loi de frottement linéaire dans la masse liquide (mouvement non turbulent) et quadratique dans la couche limite (mouvement essentiellement turbulent). La courbe intégrale sera alors d'un type intermédiaire entre le type exponentiel et le type hyperbolique, et, selon que l'un ou l'autre effet prédomine, l'allure de l'amortissement sera différente. Comme on le verra plus loin, la réalité paraît encore plus complexe et conduit en général à discriminer, $\grave{a}$ partir du fond, un film laminaire à son contact, une zone entièrement ou partiellement turbulente, éventuellement assez mince, enfin, un mouvement principal qui pourra, de nouveau, être non turbulent.

\section{c) Détermination, en laboratoire, de courbes d'amortissement de houles :}

On vient de constater que l'allure d'une courbe d'amortissement dépend de la forme de la loi de frottement admise. On passera, notamment, d'un amortissement de nature exponentielle à un amortissement de nature hyperbolique selon que cette loi est linéaire ou quadratique. Ces deux genres de courbe sont toutefois assez similaires, et leur différence ne peut s'accuser que pour un cheminement suffisamment long, la seconde amenant alors à des amortissements moindres aux grandes distances. Un mouvement ondulatoire persiste relativement plus longtemps, si la loi de frottement est quadratique. Pour des houles de laboratoire courantes, il faut disposer d'une longueur de cheminement d'une cinquantaine de mètres, sinon les différences entre les deux genres de courbe restent inappréciables, car elles sont masquées par les fluctuations inévitables du mouvement. Ces dernières représentent d'ailleurs la difficulté principale de tels essais, et il est indispensable de prendre les dispositions nécessaires pour les réduire le plus possible.

Quoi qu'il en soit, nous n'avons connaissance que d'une demi-douzaine d'essais de laboratoire permettant une discrimination suffisamment nette de la forme des courbes d'amortissement ${ }^{(1)}$. Les caractéristiques en étaient les sui-

(1) Essais réalisés en 1943 au Laboratoire Central d'Hydraulique de France. Pour l'analyse détaillée de vantes: canal rectangulaire de $120 \mathrm{~cm}$ de largeur, à fond et bords cimentés; profondeurs d'eau comprises entre 5,0 et $8,3 \mathrm{~cm}$; périodes entre 0,70 et $1,30 \mathrm{~s}$; amplitudes entre 2,5 et $0,28 \mathrm{~cm}$; nombre de points de mesure compris entre 12 et 15 répartis sur une longueur de $45 \mathrm{~m}$. Ces données correspondent à des houles longues ou pour lesquelles, en tout cas, les mouvements alternés près au fond restent notables.

Tous ces essais concordent quant à la forme des courbes d'amortissement obtenues; elles paraissent très proches d'hyperboles et, en tout cas, ne correspondent pas à des exponentielles. Il en résulte que la part prédominante des dissipations d'énergie devait répondre à une force de frottement quadratique. Notamment, de tels essais ne semblent pas conciliables avec la loi (13) correspondant à des mouvements de fluides visqueux entièrement non turbulents. Les nombres de Reynolds globaux (formule 25), relatifs à ce groupe d'essais, étaient compris entre 600 et 10.000 , selon les valeurs locales des amplitudes. Pour des écoulements permanents, ceci aurait correspondu à des régimes turbulents, car le fond n'étant pas rigoureusement lisse (on avait environ $\varepsilon=0,5 \mathrm{~mm}$ ), un nombre de Reynolds supérieur à 550 aurait déclenché la turbulence. Si l'on calcule les gradients de vitesse marginaux (formule 5) et les compare aux gradients limites d'un écoulement laminaire permanent (formule 24), on constate qu'ils étaient de 100 à 1.000 fois plus grands.

Tous ces faits se groupent en un ensemble cohérent et semblaient indiquer, sans ambiguïté possible, la présence de régimes au moins en partie turbulents. Or, certains essais récents de M. Huon Li [5], dont nous parlons au paragraphe suivant, paraissent, au moins partiellement, remettre en cause la question. En effet, selon ces expériences, tous les mouvements de houle précédents appartiendraient encore au domaine laminaire, la turbulence n'intervenant que pour des vitesses près du fond comprises entre 2 et 20 fois celles ressortant des essais.

Pour rendre compte de ces divergences, on se trouve devant l'alternative suivante : ou l'interprétation donnée aux essais ci-dessus est erronée pour une raison ou une autre et il y a lieu, en tout cas, de la soumettre à une critique attentive, ou des essais de M. Huon LI, de nature qualitative d'ailleurs, n'ont pas le sens qu'on est tenté de leur attribuer à première vue.

On va, dans ce paragraphe, examiner le premier point sous ses divers aspects :

1. Les déterminations locales d'amplitude ont résulté de moyennes étendues à un certain nom-

l'un d'entre eux, cf. [9, p. 99 et suiv.]. Les essais récents mentionnés plus loin ( $\$$ II, e) ont été effectués dans le même laboratoire. 
bre de mesures, et la précision de l'appareil limnimétrique utilisé permettait d'apprécier des amplitudes plusieurs fois plus faibles que celles obtenues. La matérialité des faits ne paraît done pouvoir être mise en cause.

2. Les amplitudes aux grandes distances de cheminement étant trop importantes pour se raccorder à une courbe exponentielle, on peut se demander si des phénomènes de réflexion en bout du canal de houle n'ont pas artificiellement accru ces amplitudes. Le canal, de $70 \mathrm{~m}$ de longueur, était terminé par une plage cimentée très peu inclinée (angle de $3^{\circ}$ ). La quote-part de houle qui aurait pu être réfléchie, dans ces conditions, serait d'amplitude micrométrique, si l'on en juge d'après une formule donnée ailleurs [8, p. 70]. Même si cette réflexion calculée était, pour une raison ou une autre, augmentée de 10 fois par exemple, elle resterait insensible. Au surplus, aucun fait expérimental en rapport possible avec un phénomène de réflexion longitudinale n'a été constaté.

3. Les fluctuations locales d'amplitude n'ont pu être entièrement éliminées. Ceci semble d'ailleurs impossible dans un canal long et étroit, dans lequel il se produit toujours certains balancements transversaux, quel que soit le soin apporté à respecter l'invariabilité de la section droite. De plus, les frottements sur les bords empêchent la réalisation de mouvements strictement cylindriques. Dans le cas actuel, les précautions suivantes avaient été prises pour réduire le plus possible l'importance de ces perturbations : réglage minutieux du rythme du batteur au cours du temps et, tout d'abord, de la direction de son axe normalement à celuj du canal; neutralisation de la partie du canal près du batteur, les premières mesures retenues se trouvant à environ 10 longueurs d'onde de ce dernier; estimation des amplitudes dans l'axe du canal. On remarquera, de plus, que les profondeurs d'eau étant faibles vis-à-vis de la largeur du canal, les perturbations dues aux bords de ce dernier ne représentaient qu'une petite fraction de celles produites par le fond, dont les essais avaient justement pour but de déceler l'influence. Il semble done que l'état cinématique du mouvement, c'est-à-dire la loi d'amortissement des amplitudes, n'ait pu être notablement modifié par la largeur finie du canal et que, d'autre part, Ia prise en compte de valeurs moyennes pour les amplitudes a éliminé, au moins en partie, l'incidence des balancements transversaux. Il n'en reste pas moins que la courbe effective des amplitudes, au lieu de s'abaisser régulièrement au fur et à mesure des cheminements, présente des fluctuations, ce qui ne permet pas un contrôle absolument strict de la loi d'amortissement théorique. Toutefois, comme on l'a si- gnalé, des courbes hyperboliques représentent, à fort peu près, la meilleure approximation, et ceci a été confirmé, dans deux cas, par un calcul basé sur la méthode des moindres carrés.

En résumé, il paraît peu probable que les essais relatés ci-dessus aient pu conduire à des conclusions erronées. Cependant, leur nombre apparait faible, et leur domaine trop étroit quant aux profondeurs d'eau et aux périodes. Eu égard, en outre, aux résultats de M. Huon $L I$, il serait très désirable que de nouveaux essais servent de contrôle aux précédents. IIs nécessiteraient, pour aboutir à des conclusions vraiment définitives, un canal d'une longueur utile de 50 à $100 \mathrm{~m}$, assez large (quelques mètres au moins), de section et de nature de fond rigoureusement constantes, un batteur muni des derniers perfectionnements et, de plus, des filtres ou dispositifs souples disposés devant le batteur afin de régulariser la houle, et à l'extrémité du canal pour empêcher toute réflexion. Le vœu que nous émettons risque alors d'être assez longtemps platonique, car de tels essais seraient dispendieux et ne pourraient, par exemple, être couplés avec ceux nécessités par l'étude de maquettes de ports, pour lesquels une longueur de cheminement d'une trentaine de mètres est ordinairement suffisante ${ }^{1}$ ). Ils vaudraient néanmoins la peine d'être tentés, afin d'élucider complètement, d'une manière quantitative et difficilement contestable, le problème de l'amortissement spatial des houles.

\section{d) Recherches de M. Huon Li sur la stabilité des couches limites oscillantes [5] :}

M. Huon Lr s'est proposé l'étude, par observation visuelle, des phénomènes de transition entre le régime laminaire et le régime turbulent, produits par un mouvement ondulatoire de l'eau dans la couche limite oscillante au contact du fond. Comme il entendait couvrir tout le domaine des houles naturelles, il a dû utiliser un appareillage particulier et a, en réalité, examiné un cas un peu différent, l'eau restant en principe immobile et le fond du bac, dans lequel elle se trouvait, étant animé de mouvements de va-etvient.

Avant de donner un compte rendu sommaire de ces essais et des conclusions à en tirer, quelques mots sur la turbulence et les couches limites ne seront pas inutiles.

Dans un écoulement turbulent permanent, par exemple dans une conduite supposée lisse, on distingue, au contact de la paroi, un film laminaire à très fort gradient, la vitesse passant vraisemblablement de zéro à la paroi à une valeur proche de la vitesse moyenne sur une

(1) Certains bassins utilisés pour l'essai des carènes pourraient toutefois convenir. 
distance ordinairement très inférieure $\grave{a}$ un millimètre. Le complément ae l'écoulement est turbulent. Le film laminaire, imposé par la condition limite à la paroi, subsiste toujours, quelle que soit la vitesse moyenne, mais son épaisseur décroît environ comme l'inverse de celle-là. Si la paroi est rugueuse, les aspérités étant toutefois assez faibles pour rester nettement noyées dans le film laminaire, les caractéristiques du mouvement changent peu; par contre, si la dimension des aspérités est du même ordre ou plus grande que l'épaisseur du film laminaire qui s'établirait en leur absence, la condition limite est profondément modifiée. Des mouvements tourbillonnaires se détachent des aspérités et des zones d'eau morte, également tourbillonnaires, se forment entre elles. En fait, le fluide se comporte comme s'il n'adhérait plus à la paroi, sa vitesse moyenne au contact de celle-là, c'est-à-dire sur la surface adoucie enveloppant les aspérités, étant déjà notable et souvent à peine inférieure à la vitesse moyenne de l'écoulement. Comme l'épaisseur du film laminaire décroît théoriquement sans limite si la vitesse augmente suffisamment, tous les écoulements turbulents assez rapides (à très grand nombre de Reynolds) répondent aux conditions précédentes. Autrement dit, toute paroi, même très lisse, finit dans ce cas par se comporter comme si elle était rugueuse.

Si le mouvement correspond à des conditions localement variables, des phénomènes annexes et transitoires apparaissent, car l'écoulement ne peut s'adapter instantanément à de nouvelles données. Prandtr (cf. notamment [1, p. 54] a, dans ce cas, introduit la notion de couche limite (1). Partons, par exemple, d'un mouvement initialement laminaire à vitesse de base constante au cours du temps, mais modifions, dans l'espace, les conditions limites. Il pourra s'agir d'une plaque immergée dans le liquide ou d'un ajutage débouchant d'un réservoir. A partir de l'arête antérieure de la plaque ou du début de l'ajutage, se développe une couche limite d'épaisseur croissante, dans laquelle le gradient est très élevé, la vitesse passant de zéro à la paroi jusgu'à sa valeur de base. Si cette dernière est assez grande, l'écoulement dans la couche limite finira par devenir turbulent. Dans ces conditions, en s'éloignant de la paroi, on constatera la présence :

a) du film laminaire très mince à fort gradient,

b) d'une couche limite turbulente,

c) d'un mouvement principal qui, primitivement laminaire, deviendra turbulent par « contagion ». Ce schéma d'écoulement correspond à une paroi lisse; si elle est

(1) Ce terme a une acception un peu différente de celle utilisée habituellement (cf. $\$ 1^{\circ}$ ). rugueuse, on retrouvera, dans le domaine du film laminaire, les phénomènes décrits plus haut.

Le type de monvement transitoire qui entre en ligne de compte dans le cas des houles est apparenté au précédent, mais non identique. Dans ce cas, les conditions limites de nature spatiale restent invariables (fond continu de rugosité donnée); par contre, les conditions au cours du temps (les vitesses du mouvement principal) sont variables. A cet égard, M. Huon LI a fait une courte relation des principaux faits antérieurement connus, résultant des observations de divers expérimentateurs et des siennes, sur le comportement des couches limites (non oscillantes) répondant à une gamme de vitesses croissantes. Le mouvement de base étant initialement laminaire, il fait ressortir l'apparition dans la couche limite, à partir d'une certaine vitesse, de «bouffées» turbulentes intermittentes, dont les intervalles au cours du temps diminuent si la vitesse augmente, et qui, tout d'abord, tendent à se diffuser dans la masse liquide en mouvement laminaire, tout en laissant subsister des trâ̂nées turbulentes. Si la vitesse augmente suffisamment, ces dernières, de plus en plus nombreuses, finissent par constituer une couche turbulente continue d'épaisseur plus ou moins grande et pouvant, éventuellement, englober toute la masse liquide. Cette description, qui ressort notamment des observations et des idées de Emmons [4], nous paraît fournir la clef du problème des couches limites oscillantes, ainsi qu'on le verra plus loin.

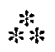

Partant de l'idée très généralement admise que les afrouillements sur fonds sableux, dus à l'action de la houle et constatés jusqu'à $20 \mathrm{~m}$ de profondeur au moins, résultent de phénomènes de turbulence près du fond, M. Hoon Li a entendu couvrir, dans ses expériences, tout le domaine des houles naturelles dont les périodes, au sens large, s'échelonnent de 0,4 à $60 \mathrm{~s}$, et les amplitudes de $0,15 \mathrm{~m}$ à $3 \mathrm{~m}$ et plus. Pour ce faire, l'auteur a cherché à réaliser l'équivalent des mouvements marginaux correspondants, en utilisant un bac contenant de l'eau et vitré, de $3,60 \mathrm{~m}$ de long, $0,90 \mathrm{~m}$ de haut et $0,30 \mathrm{~m}$ de large, dont le fond métallique, rendu mobile sur une surface de $1,80 \times 0.290 \mathrm{~m}$, était soumis à des déplacements horizontaux alternés, répondant sensiblement à une loi sinusoïdale au cours $\mathrm{du}$ temps, d'une amplitude comprise entre 6,3 et $122 \mathrm{~cm}$, s'effectuant à une cadence de 1 à 150 va-et-vient par minute. La surface de la partie mobile du fond était, soit lisse (cire coulée sur la surface), soit rugueuse de deux types : 
a) Rugosité bi-dimensionnelle représentée par des barres de bois transversales accolées, de section semi-circulaire, dont le rayon (la hauteur) était compris entre 9,5 et $32 \mathrm{~mm}$;

b) Rugosité tri-dimensionnelle constituée d'une couche de sable ou de gravier, vraisemblablement fixée sur le fond, et de dimensions $0,275,0,94$, ou $13,8 \mathrm{~mm}$.

Les essais ont consisté à déverser dans l'eau des cristaux de permanganate de potasse et à observer les phénomènes près du fond. En faisant abstraction d'une certaine difiusion moléculaire, les traces rouges laissées par la chute des cristaux vers le fond du bac restaient assez longtemps nettes et sensiblement fixes jusqu'à une distance de l'ordre de 5 à $6 \mathrm{~mm}$ du fond par exemple. Plus bas, c'est-à-dire dans le domaine proche de celui de la couche limite, elles commencaient à osciller. Si la fréquence des va-etvient était faible, un nuage assez dense de sel dissous, comportant toutefois des traînées horizontales dans le sens inverse des déplacements $\mathrm{du}$ fond, se formait ensuite sur une épaisseur de 5 à $6 \mathrm{~mm}$ en moyenne. En accroissant progressivement la fréquence, ces traînées se mettaient à onduler, et se dissipaient, indiquant ainsi le développement de la turbulence marginale. La fréquence et l'élongation correspondantes du mouvement du fond mobile étaient adoptées cornme définissant le passage du régime stable au régime instable. Toutefois, la transition ne semblait pas très fixe, car la dispersion des résultats, pour des expériences identiques, était notable.

Pour Jes fonds rugueux, on constatait, en outre, mème pour des frécfuences faibles, des tourbillons à l'aval des aspérités, dont le sens se renversait périodiquement. Ce critère n'était pas retenu comme définissant la turbulence, mais bien le fait que, pour une certaine fréquence plus forte, ces tourbillons paraissaient se détacher du fond et diffuser dans la masse liquide.

Les résultats obtenus montrent que le critère d'instabilité dépend nettement de la nature du fond. Toutefois, une surface lisse ne répond pas à la réalité expérimentale (1), ni d'ailleurs la rugosité assez artificielle dite bi-dimensionnelle

(1) Au surplus, M. Huon Li a constaté que, pour du sable de dimension $\varepsilon=0,275 \mathrm{~mm}$, le fond ne pouvait se comporter comme étant lisse que si l'épaisseur de la couche limite était 6,6 fois plus grande au moins. Aucune houle de période inférieure à $10 \mathrm{~s}$ ne répond à ce critère selon la formule (4), et toutes les houles sans exception doivent être considérées comme se propageant sur fond rugueux dès que $\varepsilon>0,5 \mathrm{~mm}$, cette dernière valeur correspondant, à peu près, aux essais courants de laboratoire. et I'on pourra, ci-après, se borner à considérer les rugosités tri-dimensionnelles (du type sable ou, par assimilation, sol cimenté). Les essais correspondants de M. Huon LI, assez fortement extrapolés, ont été condensés par M. Valembors [11] sous forme d'abaques portant, par exemple, en ordonnée, les vitesses maxima $u_{m}$ près du fond et, en abscisse, les périodes. Pour chaque rugosité $\varepsilon$ correspond un domaine de stabilité et un autre d'instabilité. D'une façon générale, les vitesses critiques diminuent lorsque la rugosité augmente.

$$
*
$$

I.es recherches précédentes appellent les commentaires suivants :

1. Il s'agit d'essais d'ordre qualitatif, limités à l'estimation de la nature cinćmatique des mouvements. Les critères adoptés apparaissent même quelque peu subjectifs, quoique le grand nombre des résultats et leur aspect homogine leur confèrent, sans doute, une signification assez précise. Encore, faut-il bien définir laquelle.

2. Les critères en question se rapportent essentiellement à la seule conche limite. En effet, avec le genre d'expériences de M. Huon Li, les mouvements de la masse liquide restent, en principe, insensibles hors de cette couche et ne peuvent conduire à des dissipations d'énergie appréciables; de toute facon, ils seraient non turbulents. Sous une autre forme, le paramètre de rugosité (27) étant mis à part, ils ne peuvent dépendre que du seul nombre de Reynolds marginal (26) et, par conséquent aucunement de la profondeur de l'eau (1.) ou de ce que nous avons nommé le nombre de Reynolds global (25).

3. Si l'on admet, comme le fait implicitement l'auteur, la transposition des résultats au phénomène de la houle réelle, on est conduit à schématiser de la facon suivante les mouvernents produits (cf. M. VAJ.EMBors [11]) : dans le domaine de stabilité, la houle est portée par une couche laminaire (la masse liquide serait, en totalité, non turbulente); dans le domaine d'instabilité, la houle est portée par une couche limite turbulente $\left({ }^{2}\right.$ ) (le complément étant, en principe, non turbulent). Les abaques de M. Valembors montrent alors que la houle naturelle serait assez souvent portée par une couche limite laminaire et les

(1) Dans la limite de la précision obtenue, les résultats n'ont effectivement pas varié, lorsque la profondeur était modifiée dans le rapport 1 à 5 environ.

(2) On fait abstraction du film laminaire devant subsister au contact immédiat du fond. 
houles de laboratoire en majorité (1). O'n se trouverait donc en contradiction avec les essais d'amortissement de houle relatés plus haut $(\S I I, c)$, car ceux-ci ne peuvent, apparemment, correspondre à des mouvements entièrement non turbulents.

La première idée qui vient à l'esprit, pour expliquer cette divergence, est de la relier à la nature des essais de M. Huon LI, ne faisant intervenir que le seul nombre de Reynolds marginal. Poussons les choses à l'extrême dans l'autre sens en considérant une houle dans la zone du fetch: elle déferlera, et toutes les couches supćrieures de l'eau seront turbulentes; d'autre part, l'eau étant profonde, les déplacements près du fond auront des élongations micrométriques; on se trouverait donc nécessairement dans le domaine de stabilité et, pourtant, le mouvement serait partiellement turbulent. On est donc amené à s'interroger sur les points suivants:

a) Ia suppression de tout mouvement ondulatoire dans les essais de M. Hron Li n'ac-croît-elle pas de façon artificielle le domaine de stabilité?

b) Quels sont les rôles respectifs joués par les nombres de Reynolds global et marginal dans la fixation des caractéristiques cinématiques des mouvements de houles?

Remarquons d'abord que la théorie de la couche limite indiquée au début de cette note est basée sur l'équation simplifiée (1), faisant abs-

(1) Ces constatations supposent l'identité entre la vitesse $u_{m}$ donnée par les essais de M. Huon Lx et celle, marginale, de la houle correspondante, telle qu'elle se produirait dans un fluide parfait. Cette assimilation n'est pas évidente dans tous les cas.

Tant que le mouvement ondulatoire reste entièrement non turbulent, les vitesses, même très près du fond, c'est-à-dire à une distance égale à un petit multiple de $z_{0}$, sont essentiellement celles produites dans un fluide parfait (cf. fig. 2). Dans la zone strictement marginale, les vitesses, à partir du fond, croissent presque linéairement jusqu'à la distance $z_{0}$, puis, après quelques oscillations très rapidement amorties, se raccordent à la vitesse $u_{m}$ du fluide parfait. Cette dernière peut donc, valablement, être identifièe à celle des oscillations du fond dans les essais de M. Huon Lr.

Il est peu probable, si la conche limite n'est pas entièrement laminaire, que le diagramme des vitesses ait l'allure ci-dessus. Il semble que ces vitesses devraient passer plus progressivement à la vitesse $u_{m}$; autrement dit, Ia zone de raccordement serait plus épaisse et, sans doute, variable selon l'intensité de la turbulence. L'expérience n'a pas encore complètement tranché la question, semble-t-il, et les opinions diffèrent. Pour les uns, la vitesse "près du fond (sans autre précision) serait de l'ordre de $u_{m}$, pour les autres, inférieure d'un certain pourcentage. Quoi qu'il en soit, il n'est pas sûr que dans le domaine d'instabilité, on puisse identifier, sans précaution, la vitesse de M. Huon Lr ou, plus généralement, celle dite « près du fond », avec celle, marginale, du fluide parfait en mouvement ondulatoire. C'est traction des termes quadratiques dus aux accélérations de la masse liquide. Autrement dit, l'effet des brassages alternés de l'eau n'y figure prs. Quoique ceci semble avoir peu d'importance dans la limite d'application des équations de Navier (la théorie rigoureuse de M. Carry ne conduit pas à des résultats essentiellement différents), le déclenchement des phénomènes d'instabilité peut, par contre, être sérieusement influencé par les alternances de courant et, dans l'état actuel des connaissances théoriques, l'expérience seule paraît pouvoir en décider. Un contrôle direct en mer couvrant le domaine exploré par M. Huon Lr, c'est-à-dire jusqu'à des périodes de houle de $20 \mathrm{~s}$ par exemple, est visiblement exclu ou nécessiterait des moyens matériels vraiment hors de proportion avec les résultats à en attendre. Reste l'emploi de canaux expérimentaux, qui permettent, aujourd'hui, la réalisation de mouvements ondulatoires ayant jusqu'à plusieurs secondes de période, et correspondant déjà à des houles naturelles. Quelques contrôles dans le domaine des périodes comprises entre 0,4 et $4 \mathrm{~s}$ par exemple, devraient déjà donner des indications suffisantes, eu égard à la nature des renseignements cherchés.

Quoi qu'il en soit, nous avons estimé intéressant de procéder à un premier contrôle de cette espèce, en vue d'obtenir un apercu de la question et d'orienter de nouvelles recherches, dans la limite où elles apparaîtraient utiles. Malgré son caractère fragmentaire, qualitatif et quelque peu subjectif, puisqu'il s'agit de l'interprétation d'observations visuelles, ce groupe d'essais est instructif, et permet, semble-t-il, de clarifier quelques grandes lignes du probleme.

\section{e) Examen de la zone marginale d'une houle de laboratoire :}

Les essais ont été réalisès dans un canal de houle vitré de $100 \mathrm{~cm}$ de largeur et $50 \mathrm{~m}$ de longueur pour une profondeur d'eau $H$ de $44 \mathrm{~cm}$ (période $2 \mathrm{~T}=2,04 \mathrm{~s}$, longueur d'onde $400 \mathrm{~cm}$ ). Les ondes pouvaient être considérées comme longues, les mouvements marginaux restant importants. La rugosité $d u$ fond correspondait à $\varepsilon=0,5 \mathrm{~mm}$ environ (sol cimenté) et, sur une partie, à $\varepsilon=10 \mathrm{~mm}$ (couche de gravillon). La houle était régularisée (générateur à éléments souples) et la réflexion de la plage d'extrémité annulée par des dispositifs amortisseurs. De très petits cristaux de permanganate de potasse étaient déversés en divers points proches du plan médian du canal. Les observations concernant les couches profondes de l'eau, à partir du moment oì les cristaux avaient atteint le

toutefois ce qu'on fait habituellement faute de micux. (Notamment, il a ćté procédé de cette façon pour les houles consignées au tableau I ci-après). 
fond, s'étendaient sur trois minutes environ. Trois amplitudes de houle de $6,0-1,5$ et $0.4 \mathrm{~cm}$ ont été utilisées. Les résultats pour les deux premières figurent au tableau I. aurait dû, par conséquent, être laminaire et l'ensemble du mouvement non turbulent. Or, autour de chaque grain de permanganate reposant sur le fond, on constate, apparemment à cadence

\section{TableaU I}

Comportement de la zone marginale d'une houle de laboratoirc.

Date : $5-7-56$

Température de l'eau : $18^{\circ}$

Coefficient de viscosité cinématique $v=1,11 \times 10^{-2} \mathrm{~cm}^{2} / \mathrm{s} ; z_{0}=0,84 \mathrm{~mm}$

(formule 4)

\begin{tabular}{|c|c|c|c|c|c|c|c|c|}
\hline \multirow{4}{*}{$\begin{array}{c}\text { Amplitude } \\
2 h \\
\mathrm{~cm}\end{array}$} & \multirow{2}{*}{$\begin{array}{c}\text { Vitesse } \\
\text { maximum } \\
u_{m}\end{array}$} & \multirow{2}{*}{$\begin{array}{c}\text { Excursion } \\
\text { maximum } \\
2 r\end{array}$} & \multirow{4}{*}{$\begin{array}{l}\text { Nombre } \\
\text { de } \\
\text { Reynolds } \\
\text { global }\end{array}$} & \multicolumn{4}{|c|}{ TYPE DE RUGOSITÉ } & \multirow{4}{*}{ REMARQUES } \\
\hline & & & & \multicolumn{2}{|c|}{$\varepsilon=10 \mathrm{~mm}$} & \multicolumn{2}{|c|}{$\varepsilon=0,5 \mathrm{~mm}$} & \\
\hline & \multicolumn{2}{|c|}{ près du fond } & & & & & & \\
\hline & $\mathrm{cm} / \mathrm{s}$ & $\mathrm{cm}$ & & $u_{m} / u_{o}$ & $z_{t} / z_{0}$ & $u_{m} / u_{c}$ & $z_{1} / z_{0}$ & \\
\hline 6,0 & 12,5 & 8,2 & 53.000 & 1,25 & 105 & 0,42 & 35 & Turbulence complète \\
\hline \multirow[t]{2}{*}{1,5} & 3,10 & 2,0 & 13.200 & 0,31 & 35 & 0,10 & 12 & Turbulenceincomplète \\
\hline & (8) & (9) & $(25)$ & & & & & $N^{\circ}$ de formule \\
\hline
\end{tabular}

$u_{c}$ représente la vitesse de transition ou critique résultant des essais de M. Huon Li et tirée des abaques de M. Valembois $\left(u_{c}=10 \mathrm{~cm} / \mathrm{s}\right.$ pour $\varepsilon=10 \mathrm{~mm} ; u_{c}=30 \mathrm{~cm} / \mathrm{s}$ pour $\left.\varepsilon=0,5 \mathrm{~mm}\right)$. $z_{t}$ est la moyenne, ressortant de plusieurs observations, de l'épaisseur de la couche de fond à l'état entièrement ou partiellement turbulent. La turbulence a été dénommée complète lorsque, au cours du temps dévolu à l'essai, il y avait diffusion généralisée et relativement homogène $\mathrm{du}$ permanganate dissous formant un nuage rose de contour d'ailleur's assez imprécis. La turbulence a été dénommée incomplète lorsqu'il subsistait, dans la zone en diffusion, des bouffées turbulentes plus ou moins distinctes. Les observations étant malaisées et à la limite de la perception pour la houle de $0,4 \mathrm{~cm}$ d'amplitude, elles n'ont pas été retenues; toutefois, les apparences étaient encore légèrement distinctes de celles d'une pure diffusion moléculaire.

D’une façon générale, les aspects du phénomène étaient moins nets et plus difficiles à apprécier pour le fond très rugueux.

Décrivons, à titre d'exemple, l'essai pour la houle de $1,5 \mathrm{~cm}$ d'amplitude avec $\varepsilon=0,5 \mathrm{~mm}$, pour lequel aucune ambiguïté quant à l'interprétation des observations n'est possible. Selon les expériences de M. Huon LI, les vitesses près du fond sont dix fois trop faibles pour créer la turbulence $\left(u_{m} / u_{v}=0,10\right)$. La couche limite double de celle du batteur, l'émission de bouffées manifestement turbulentes qui, par suite du mouvement général de l'eau (une houle comporte toujours un léger transport de masse dans un sens ou dans l'autre), se déplacent peu à peu sans se confondre d'abord, avant de diffuser progressivement et de se fondre dans le nuage général. Ce phénomène se produit à quelques millimètres du fond, et, dans le cas particulier, la hauteur du nuage diffusé est de l'ordre du $\operatorname{cm}\left(z_{t}: z_{0}\right.$ estimé égal à 12$)$.

Dans l'ensemble, les observations se résument comme suit : un film laminaire probable au contact du fond (très difficilement discernable à cause des irrégularités du fond si $\varepsilon=10 \mathrm{~mm}$, esquissé par quelques traînées très fines quasi rectilignes si $\varepsilon=0,5 \mathrm{~mm}$ ); une couche de fond au moins partiellement turbulente et plus ou moins épaisse; pour le complément de la masse liquide, un mouvement apparemment non turbulent.

Ce comportement de la couche limite oscillante n'est pas inattendu, si lon se réfère aux expériences de Emmons citées plus haut. Il résulte, en tout cas, des essais précédents une influence très sensible des oscillations de la masse liquide sur la stabilité de la couche marginale. Selon le tableau I, seule la houle pour $2 \mathrm{~h}=6 \mathrm{~cm}$ et $\varepsilon=10 \mathrm{~mm}$ devrait, d'après les expériences de M. Huon LI, être portée par une 
couche limite turbulente $\left(u_{m}: u_{c}=1,25>1\right)$. Or, pour tous les essais ci-dessus, on a constaté une turbulence au moins partielle même pour la valeur faible $u_{m}: u_{c}=0,10$. Le fait essentiel motivant ces divergences ne peut être que l'état d'accélération périodique de la masse liquide ( ${ }^{1}$ ), qui se traduit par une émission turbulente de même rythme. Un appareillage stroboscopique aurait été indiqué pour déceler si cette émission est concomittante avec les passages successifs des particules au droit de leur position de repos à la vitesse maximum $u_{n}$, ou, ce qui paraît plus probable, si les alternances de sens du courant sont responsables du fait constaté. Quoi qu'il en soit, il semble qu'il faille répondre affirmativement à la première question posée au paragraphe d) précédent. Fn la retournant, on pourra lui donner la réponse suivante : la présence d'un mouvement ondulatoire dans la masse liquide diminue de façon notable la stabilité de la conche limite, telle qu'elle ressort des essais de M. Huon Lr.

Examinons maintenant l'épaisseur de la couche turbulente. Selon le tableau I, elle paraît croître de façon plus ou moins linéaire avec la vitesse $u_{m}$ et, par conséquent, avec le nombre de Reynolds global $\mathcal{R}_{1}$ indiqué, ou avec le nombre marginal $\mathcal{R}_{2}$, également proportionnel à $u_{m}$. D'autre part, elle croît aussi, mais dans une proportion plus faible, avec la rugosité $\mathrm{d} u$ fond. Pour le cas correspondant, à peu près, à la valeur critique de M. Huon LI, cette couche est déjà fort épaisse :

$$
\left(z_{t}=105 z_{0}=105 \times 0,084=9 \mathrm{~cm}\right) .
$$

Ce fait est également à mettre à l'actif des oscillations d'ensemble de la masse liquide, car, selon les essais de l'auteur en question, cette couche devrait être d'épaisseur beaucoup plus faible (un très petit multiple de $z_{0}$ au plus). Il a visiblement comme consćquence des dissipations d'énergie par turbulence nettement plus grandes que celles qui se produisent avec fond oscillant et masse liquide immobile $\left({ }^{2}\right)$. Les houles consignées sur

(1) On observera toutefois que Ies critères de M. Huon $\mathrm{LI}_{\mathrm{I}}$ se rapportent à une turbulence importante; il ne paraît pas avoir été noté la gradation que nous avons observée et qui conduit d'une turbulence "incomplète 》 à une turbulence « complète»; peut-être n'existe-t-elle pas avec le mode opératoire utilisé ou est-elle très brève?

(2) En théorie, les essais de M. Huon Lr auraient pu être couplés avec des mesures de perte d'énergie dans la masse liquide, déduites de la puissance absorbée par le mécanisme entrainant le fond. Il est douteux, cependant, qu'on puisse, de cette facon, arriver à discriminer nettement l'énergie dissipée dans l'eau (les pertes par frottement mécanique, celles, anormales, intervenant sur le pourtour et au droit des attaches de la partie mobile du fond, l'énergie dépensée à créer les légères oscillations stationnajres parasites constatées, etc., représentent, sans doute, la majorité de l'énergie absorbée). le tableau I étant longues, il n'y aurait pratiquement rien de changé quant aux valeurs de $z_{t}$ si $2 \mathrm{~T}$, c'est-à-dire $z_{0}$, restant fixes, la profondeur $H$ était fortement diminuée, l'amplitude étant réduite dans le rapport $\sqrt{\mathrm{H}}$, afin de conserver les mêmes valeurs $u_{m}$. Dans ces conditions, et selon l'essai envisagé, une part notable, ou même la totalité de la tranche liquide auraient été à l'état turbulent, et ceci d'autant plus que les valeurs $z_{t}$ indiquées sont des minima risquant d'être notablement dépassés dans la plupart des cas courants, c'est-à-dire, notamment, pour des houles moins pures que celles anaIysées ( ${ }^{3}$ ).

La réponse à la seconde question posée plus haut : rôles respectifs à attribuer aux nombres de Reynolds $\mathcal{R}_{1}$ et $\mathcal{R}_{2}$ pour la fixation de la nature cinématique des mouvements ondulatoires, ne ressort que d'une façon fragmentaire des essais précédents. Il serait nécessaire, pour pouvoir conclure d'une manière précise, d'étendre systématiquement leur domaine. On devra donc se borner, pour l'instant, à des indications d'ordre général.

Supposons la période 2 'T fixe et envisageons, comme paramètre de comparaison, la composante moyenne maximum de la vitesse, soit $\bar{u} \bar{u}_{m}$. On aura toujours $u_{m} \leqslant \bar{u}_{m}$, l'égalité valant pour les ondes longues, c'est-à-dire pour le domaine de l'eau peu profonde. Maintenons d'abord les valeurs $H$ assez faibles pour répondre à cette hypothèse. Dans ces conditions, pour un $u_{m}$ donné. le nombre de Reynolds marginal $\boldsymbol{\alpha}_{2}$ est indépendant de la profondeur, tandis que le nombre global $\mathfrak{R}_{1}$ lui est proportionnel [formules (25) et (26)]. Lorsque $u_{m}$ crô̂t, le déclenchement initial de la turbulence, qui sera a priori inccmplète, doit correspondre à $z_{t}$ petit; ce critère d'instabilité est visiblement indépendant de la profondeur et sera commandé par $\mathcal{R}_{2}$, comme dans les essais de M. Huon Li (*). Toutefois, la valeur critique de ce paramètre doit être nettement plus faible, si l'on en juge d'après les

(3) Comme causes très probables d'accroissement des valeurs effectives de $z_{t}$, on peut citer: 1) le maintien des mouvements ondulatoires durant un temps ordinairement bien plus long que celui adopté pour les essais; 2) la densité supérieure à celle de I'eau des nuages chargés de permanganate dissous, ce qui contrarie leur diffusion dans le sens vertical; 3) la nature moins régulière des houles correspondant aux conditions courantes d'application. Même les houles utilisées pour les études de maquettes de ports et, a fortiori. les houles naturelles, ne peuvent se comparer aux houles de canal filtrées, telles qu'on peut les produire aujourd'hui rn laboratoire.

(4) Sur fond absolument lisse, les essais de M. Huow Is font ressortir un nombre marginal crifique 6 s? de $800: \sqrt[2]{2}=566$, en bon accord avec le nombre critique $\mathscr{R}_{\bullet}=550$ (formule 23) dérivé, pour le cas d'écoulements permanents sur un plan, des essais de tubes circulaires lisses. 
essais de contrôle précédents. $u_{m}$ croissant toujours, la turbulence complète se produira dans la couche marginale avec une épaisseur $z_{t}$ qui pourra être inférieure ou égale à $H$. Dans le premier cas, $\mathcal{R}_{2}$ sera toujours déterminant pour cette nouvelle phase d'instabilité; dans le second, $\mathcal{O}_{1}$ entrera en ligne de compte, puisqu'il dépend de $\mathrm{H}$.

Considérons maintenant le domaine de l'eau assez profonde relativement proche de l'eau profonde, c'est-à-dire, augmentons $H$ suffisamment pour que la vitesse $u_{m}$ près du fond soit, sinon insensible, du moins très faible. Il ressort alors d'essais d'amortissement de houles signalés ailleurs $[9$, p. 97 et suivantes], que les dissipations d'énergie correspondent à des régimes au moins partiellement turbulents, lorsque le nombre de Reynolds global $\mathcal{R}_{1}$ dépasse environ 1.000 (des valeurs plus faibles n'ont pu être analysées, car les houles étaient trop faibles pour répondre aux techniques courantes de laboratoire). On constate, en effet, que si ces mouvements avaient été entièrement non turbulents, la dissipation d'énergie dans la masse liquide due à la viscosité [quote-part $Q_{1, v}$ formule (15)? serait, environ, dix fois trop faible pour expliquer les amortissements observés et que, d'autre part, cette dissipation d'énergie dans la couche limite [quote-part $Q_{2,}$ formule (17)], serait également beaucoup trop faible, à cause de la petitesse de $u_{m}$. Par la même occasion, $\boldsymbol{\alpha}_{2}$ est pratiquement négligeable. En résumé, pour ces natures d'essais il $\mathrm{y}$ a turbulence au moins partielle, et comme elle ne peut dépendre du nombre marginal $\mathfrak{R}_{2}$, c'est le nombre global $\mathfrak{R}_{1}$ qui doit intervenir ( ${ }^{1}$ ).

Dans le domaine intermédiaire entre les deux précédents, le rôle prédominant pour le déclenchement de la turbulence, lorsque la profondeur relative croîtra, sera tenu, successsivement, par $\mathfrak{\mathcal { R }}_{2}$, puis par $\boldsymbol{\mathcal { R }}_{1}$.

Les essais d'amortissement de houle dont il est question ci-dessus se référaient, comme indicateur de turbulence, au seul nombre de Reynolds global $\boldsymbol{\alpha}_{1}$. La discussion plus détaillée précédente montre que si ce point de vue est justifié dans le domaine de l'eau assez profonde ou profonde, il est indiqué, dans le domaine des houles longues, de donner la primauté au nombre marginal, du moins tant qu'il s'agit de fixer le déclenchement initial des régimes non laminaires.

Il semble au surplus qu'au stade actuel de la qustion, l'emploi du nombre $\mathcal{R}_{1}$, en relation avec la valeur critique 550 concernant les écoulements permanents, fournisse, pour tous les essais courants de laboratoire en tout cas, une indication utile. On a constaté notamment que tous les essais de houle pour lesquels $\mathfrak{A}_{1}$ était supérieur à 600 , comportaient, si le cheminement était suffisant, une courbe d'amortissement sensiblement hyperbolique dénotant l'intervention d'une turbulence. D'autre part, si, selon le processus indiqué plus haut, on transpose pour une houle de laboratoire de dimensions minima celle du tableau I pour $2 \mathrm{~h}=1,5 \mathrm{~cm}$ et $\varepsilon=0,5 \mathrm{~mm}, u_{m}$ et $z_{0}$ restant fixes, on trouve pour $\mathrm{H}=2 \mathrm{~cm}$, par exemple, une amplitude réduite à $1,5 \sqrt{(2 / 44)}=0,32 \mathrm{~cm}$, et un nombre de Reynolds global $\mathcal{R}_{1}=600$.

Or, il a été constaté, dans le cas correspondant du tableau I, une turbulence au moins partielle.

\section{III. - AMORTISSEMENT DES HOULES EN PROFONDEUR FINIE ETAT ACTUEL DE LA QUESTION. - RECHERCHES A ENTREPRENDRE}

Avant de résumer dans ce paragraphe l'exposé précédent, il est utile d'examiner sommairement les buts recherchés lorsqu'on analyse les mouvements de houle dans les fluides réels et leurs modifications progressives.

Ces buts sont divers, et également les critères à prendre en compte. Sans vouloir être complet, disons que l'amortissement des houles et les phénomènes connexes intéressent :

\footnotetext{
(1) Dans le domaine de l'eat profonde, et même avant, la couche turbulente paraît se détacher du fond, au voisinage duquel les mouvements restent insensibles. La défnition du nombre global $\Re_{1}$ doit en tenir compte $[9$, page 75$]$.
}

a) Le physicien qui cherche à élucider le détail du mécanisme en contròlant ses déductions théoriques par des résultats d'essais;

b) L'hydraulicien qui désire notamment obtenir des informations précises sur les lois de similitude permettant d'extrapoler au domaine réel les observations faites sur modèles réduits;

c) L'ingénieur qui se préoccupe d'estimer l'amortissement des houles du large dans' les régions côtières ou portuaires et, d'autre part, de recherche des critères déterminant l'érosion des fonds marins ou les transports littoraux; 
d) I'océanographe et le navigateur pour lesquels l'évolution des houles sur de grandes distances présente un intérêt particulier, en vue de prévisions à plus ou moins longue échéance.

Ce dernier aspect du problème se rapportant au domaine de l'eau profonde n'est pas en rapport direct avec le sujet traité, et l'on se contentera de cette courte mention.

$$
\text { **: }
$$

a) Pour le théoricien, la question se présenterait de façon en somme assez simple, quelle que soit la profondeur relative de l'eau, si les mouvements étaient entièrement non turbulents. L'étude approchée de la couche limite, faite au début de cette note, ou, si l'on recherche la rigueur, la solution de M. CARRY permettent, pour des régimes établis et des fonds peu variables, de prévoir des courbes exponentielles d'amortissement des amplitudes, dont le décrément comporte un terme principal proportionnel à $v^{1 / 2}$, c'est-à-dire relativement grand. Ce terme, dû à la forte concentration des dissipations d'énergie dans la couche limite marginale à gradient de vitesse très élevé (on admet une vitesse nulle au contact du fond), disparait en même temps que cette couche, si l'eau est profonde, car les vitesses près du fond sont alors négligeables. On fera dorénavant abstraction de ce dernier cas.

Les calculs précédents apparaissent toutefois illusoires la plupart du temps et pour diverses raisons :

1. En ce qui concerne les mouvements de houle, tous les fonds naturels et de laboratoire doivent être considérés comme rugueux; ceci ressort, par exemple, des essais de M. Huon Lr signalés plus haut (le genre particulier de ces expériences n'a pas de répercussion dans ce cas). Il en résulte que la condition limite de vitesse nulle à la paroi perd, en somme, sa signification par suite de remous tourbillonnaires sur le fond; notamment, les valeurs pondérées des vitesses, au niveau des aspérités du fond, sont déjà très notables.

2. Si les alternances de courant sont assez lentes pour être négligeables, le mouvement est assimilable à un écoulement permanent et, dans ces conditions, le très fort gradient marginal appelle nécessairement une turbulence, au moins dans les couches profondes (c'est certainement le cas pour l'onde de marée (1) et même pour les seiches).

\section{Pour toutes les houles de laboratoire (en fai-}

(1) La nature turbulente des courants de marée près des côtes a été décelée directement par l'observation des huctuations de vitesse. sant abstraction de houles quasi-micrométriques ne ressortant pas des techniques habituelles des essais) et, a fortiori, pour les houles réelles, il semble résulter de diverses constatations l'existence très probable d'une couche au moins partiellement turbulente en contact, près du fond, avec le film laminaire très mince subsistant à la paroi, et qui, parfois fort mince elle-même (pour les houles les plus faibles) peut s'étendre à une partie notable ou à la totalité de la masse liquide. Cette couche non laminaire, même mince, concentre la majorité des dissipations d'énergie et comme ces dernières, au contraire de ce qui se passe pour les écoulements visqueux, répondent à une loi de frottement non linéaire (et approximativement quariatique), la courbe d'amortissement des houles n'est plus exponentielle, mais sensiblement hyperbolique. Ce fait paraissant bien établi, et ressortant d'essais de nature quantitative concernant même des houles très faibles de laboratoire, avait tout d'abord attiré notre attention sur cet aspect du problème $[9$, p. 99 et suivantes $]$. Bien qu'il ne révèle que d'une façon indirecte le caractère au moins partiellement turbulent de tous les mouvements ondulatoires habituels, une interprétation autre que celle exposée ci-dessus paraît difficilement conciliable avec l'observation.

Toutefois, des essais plus récents de M. Huon Li [5] couvrant tout le domaine des houles réelles et de laboratoire, semblent restreindre la portée des déductions précédentes. En effet, la couche marginale turbulente se produirait bien, mais pour des vitesses près du fond nettement plus élevées que celles ressortant des courbes d'amortissement, en sorte que, pour la majorité des houles de laboratoire et une partie des houles réelles, la couche marginale conserverait son caractère laminaire. On notera cependant, au sujet de ces essais, les deux points suivants :

1. Il ne s'agit pas de mouvements effectifs de houles, mais de mouvements alternés du fond mobile d'un bac rempli d'eau restant en principe immobile.

2. Les observations sont de nature qualitative et résultent de l'appréciation visuelle du développement d'une turbulence importante au contact du fond, décelée par une diffusion rapide des traînées laissées par des cristaux de permanganate de potasse en cours de dissolution.

Afin de nous rendre compte s'il était possible de concilier les faits précédents ou, sinon, pour confirmer ces divergences, nous avons procédé à quelques essais préliminaires relatés plus haut, effectués dans un canal de houle, l'appréciation de la nature des mouvements ondulatoires réels produits ressortant également de l'utilisation de cristaux de permanganate. Les houles étaient 
soigneusement régularisées afin d'éviter tout effet parasite. Malgré leur domaine peu étendu et leur caractère qualitatif, ces essais ne paraissent guère laisser de doute quant aux faits suivants :

- La présence d'un mouvement ondulatoire effectif de l'eau augmente nettement l'instabilité de la couche marginale; autrement dit, les vitesses critiques près du fond paraissent nettement plus faibles que si l'eau est immobile et le fond oscillant;

- Le phénomène s'amorce par une turbulence vraisemblablement partielle, mais effective, et confirmée d'ailleurs par des observations antérieures d'autres expérimentateurs pour des conditions assez voisines (EMmons).

Au total, il semble que l'écart qui paraissait exister entre les deux modes de recherches précédents, courbes d'amortissement d'une part, et appréciation d'une turbulence de l'autre, est fortement amenuisé, à supposer qu'il subsistc encore.

Si l'on résume l'aspect théorique du problème, on peut dire que pour toutes les houles sensibles de laboratoire et toutes les houles réelles, il paraît se produire, au moins très près du fond et sur une épaisseur croissante avec les vitesses alternées en jeu, une couche non laminaire, partiellement ou totalement turbulente, qui rompt à peu près certainement, par suite de la nature aléatoire de ses mouvements, tont lien fonctionnel entre la couche laminaire au contact immédiat du fond et celle pouvant subsister au-dessus de la couche turbulente. Sous une forme imagée, ceci revient à supposer l'équivalent d'un arrachement foncièrement irrégulier (mais, sans modification notable de densité, ni glissement caractérisé) entre la zone intérieure de la masse liquide se déplaçant rapidement et le film laminaire contigu à la paroi. Les vitesses moyennes, dans la zone de transition turbulente, étant sensiblement celles de la partie intérieure de la masse liquide, on en déduit que, pour cette dernière, qu'elle soit à l'état turbulent ou non, l'hypothèse d'une vitesse nulle $\dot{a}$ la limite n'a plus de raison d'être. Si le mouvement de la masse liquide reste non turbulent, la courbe des vitesses doit même se comporter de façon régulière jusqu'à la limite, au droit de laquelle elle éprouvera des discontinuités de caractère aléatoire.

$b$ ) et $c$ ) Pour l'hydraulicien et l'ingénieur, la question de l'amortissement des houles et ses phénomènes annexes présente au moins deux aspects.

Lorsqu'il s'agit de fixer les lois de similitude entre un modèle réduit (à profondeurs non distordues) et le secteur réel, pour des fonds sup- posés inaffouillables, la forme des courbes d'amortissement des amplitudes entre principalement en ligne de compte. Si, dans ce cas, les résultats de M. Huon $L_{I}$ étaient définitivement confirmés pour des mouvements alternatifs effectifs, le laboratoire serait assez mal partagé, car les houles d'essai seraient portées la plupart du temps par une couche marginale laminaire, alors que les houles naturelles correspondantes le seraient souvent par une couche turbulente. II paraît bien, au contraire, d'après les essais d'amortissement de houle de laboratoire et les essais de contrôle relatés plus haut, que dans l'une et l'autre hypothèses, la loi d'amortissement est commandée par une couche marginale au moins partiellement turbulente; par conséquent, la similitude complète entre le modèle et la réalité devrait être acquise dans la grande majorité des cas.

Lorsqu'il s'agit des affouillements ou des érosions de fonds marins, le problème se pose un peu autrement, mais les faits n'infirment aucunement les opinions émises plus haut quant au déclenchement de la turbulence. M. Valembors [11] a remarqué que les débuts d'entraînement de matériaux, tels qu'ils ressortent notamment des expériences très interessantes et fort utiles de M. Larras [7], correspondraient, selon les essais de M. Huon LI, à des couches limites laminaires. Ceci paraît fort surprenant, car on lie ordinairement les phénomènes d'érosion à la turbulence ${ }^{(1)}$; au surplus, tous les fonds naturels sont à considérer comme rugueux pour les mouvements alternés et, par conséquent, le film laminaire théorique est profondément perturbé. Ces observations nous paraissent aller nettement dans le sens de celles déjà faites sur l'instabilité plus grande de la couche marginale lorsqu'il y a un mouvement effectif de houlc. La turbulence, au moins partielle, dont on a décelé l'existence dans les essais de contrôle consignés plus haut, et pour des vitesses près du fond moindres, rendrait mieux compte des faits constatés.

$$
\text { * * * }
$$

Les conclusions précédentes sur la nature cinématique des mouvements des houles réelles et de laboratoire, bien que probables à notre sens, restent cependant, pour partie, conjecturales, car les vérifications expérimentales relatées plus haut sont incomplètes. On a signalé l'intérêt de contrôles additionnels : notamment celui de la forme des courbes d'amortissement des amplitudes, couvrant un domaine plus étendu, ce qui nécessiterait, malheureusement, un canal de houle assez dispendieux; l'extension à l'ensemble du domaine réalisable en labora-

(1) C'est l'opinion de beaucoup la plus répandue parmi les théoriciens, dont M. Huon LI. 
toire (périodes de 0,4 à 4 secondes par exemple) des estimations visuelles sur la nature des mouvements de houle. Tontefois, ces derriers essais, de nature qualitative, ne seraient vraiment concluants que si on leur adjoignait un dispositif de mesure quantitatif, dont la mise au point n'apparaît cependant pas très aisée. Il serait pourtant fort désirable d'aboutir à des résultats très précis, valables dans un domaine suffisant, afin que les recherches connexes, telles que celles concernant l'amortissement des houles dans les régions côtières, les affouillements des fonds, les transports littoraux, etc., puissent être assises sur des bases indiscutables.

\section{ANNEXE \\ Calcul du taux d'amortissement d'une houle dans un fluide visqueux de profondeur constante, lorsque la vitesse se comporte régulièrement près du fond.}

L'amortissement des houles dans les fluides réels ne peut, en général, être calculé de facon rigoureuse et l'on doit se contenter de méthodes approchées. Parmi celles-ci, la plus efficace paraît être la méthode énergétique, dont la justification est avant tout d'ordre expérimental. Toutefois, il existe un cas particulier pour lequel un contrôle théorique du degré d'approximation réalisable avec cette méthode est possible, et il est intéressant de l'envisager. Il s'agit des mouvements de houle dans un fluide visqueux susceptibles d'une analyse rigoureuse. On sait $[9$, p. 51 et 64$]$ que la méthode énergétique implique, en règle générale, l'introduction, dans l'équation (11) du bilan d'énergie, des termes perturbateurs $Q$ dus aux résistances passives, évalués à partir des données du mouvement de base non perturbé (c'est-à-dire pour un fluide parfait avec vitesses non nulles sur le fond). II faut alors distinguer, en profondeur finie, d'une part la dissipation d'énergie due au mouvement répufé non turbulent à l'intérieur de la masse liquide et donnée par le terme $Q_{1, \nu}$ proportionnel $\dot{a} \vee$ (formule 15), d'autre part, la dissipation marginale d'énergie schématisée habituellement par une force représentative de frottement et calculée également en fonction des vitesses du mouvement de base. On a vu d'ailleurs que pour la presque totalité des cas, cette dernière dissipation d'énergie est la plus importante (en eau peu profonde du moins) et correspond à des phénomènes de turbulence.

Quoi qu'il en soit, l'évaluation rigoureuse du terme à comparer à $Q_{1, v}$ doit être basée sur un mouvement de houle dans un fluide visqueux avec vitesse non nulle sur le fond ( ${ }^{1}$ ). Il semble donc, à première vue, que le contrôle en question, se bornant à une justification de la méthode énergétique, soit d'un intérêt surtout théorique.

(1) Plus exactement, il ne doit être imposé aucune condition particulière à la composante horizontale de la vitesse marginale; autrement dit, cette dernière doit sc comporter régulièrement près du fond.
En fait, son incidence pratique est fort loin d'être négligeable. On a constaté en effet, au paragraphe III précédent, que tous les mouvements ondulatoires assez peu accusés pour correspondre à un régime essentiellement non turbulent à l'intérieur de la masse liquide, doivent comporter néanmoins, même pour les houles faibles de laboratoire, une couche marginale non laminaire, éventuellement fort mince, qui interrompt, par ses impulsions de caractère aléatoire, la distribution interne des vitesses. Ces dernières seront donc non seulement différentes de zéro "sur le fond », c'est-à-dire au contact de la zone marginale, mais se comporteront a priori de façon régulière dans son voisinage immédiat.

Le calcul rigoureux des houles dans un fluide visqueux, basé sur les hypothèses précédentes, est assez complexe et avait été esquissé seulement dans [9 p. 93]. Toutefois, on pourra le présenter sous une forme relativement condensée, car la plupart des calculs publiés notamment par M. Carry [3], dont on conservera les notations, sont transposables au cas actuel. Indiquons d'abord, de façon précise, le résultat auquel on aboutit :

Si la vilesse n'est pas nulle sur le fond et s'y comporte d'une façon réguliere, le taux d'amortissement spatial d'une houle en profondeur. constante dans un fluide visqueux, a comme valeur principale un tcrme en $v$ et non en $v^{1 / 2}$, identique à celui donné par la formule (13) lorsque $\mathrm{Q}$ a la valeur $\mathrm{Q}_{1, \nu}$, les termes négligés étant des ordres $v^{3 / 2}$ et plus.

Cet énoncé apporte le contrôle cherché du mode de calcul énergétique utilisé, puisque les termes principaux sont identiques dans les deux cas, les différences n'intervenant que pour des termes d'ordre ${ }^{3 / 2}$, extrêmement petits en général ( ${ }^{(1)}$.

(1) Ce résultat signifie que, mis à part la couche marginale, je mouvement ondulatoire d'un fluide visqueux diffère très peu de celui d'un fluide parfait. Cette conclu- 
***

La fonction du courant II correspondant au problème peut être admise identique à celle utilisée par M. Carry. C'est d'ailleurs le type de solution de Basset, déjà adopté par M. BıESEL [2].

Cette fonction concerne des « petits mouvements » et s'écrit, pour une houle progressant en direction des $x$ positifs, la valeur $z=0$ correspondant maintenant au niveau de l'eau au repos :

$\Psi=(\mathrm{A} \operatorname{ch} \alpha z+\mathrm{B} \operatorname{sh} \alpha z+\mathrm{C} \operatorname{ch} \beta z$

$$
+\mathrm{D} \operatorname{sh} \beta z) e^{-i(a+i b t}
$$

où :

$$
\beta^{2}=\alpha^{2}+\frac{i b}{v}
$$

Les parties réelles de $\Psi$ et de ses dérivées sont seules à prendre en compte.

La constante $\alpha$, de l'ordre de grandeur de $a$ [formule (6)] doit être considérée comme une quantité finie, de même que la constante $b$ [formule (2)] à choisir réelle pour un régime établi; par contre, la constante $\beta$ est très grande d'après (29), car v est très petit; le développement de $\beta$ en puissances croissantes de y débute par un terme principal en $v^{-1 / 2}$, et l'on peut supposer sa partie réelle positive.

Lorsque $x$ croît, la variation des amplitudes, à l'approximation des petits mouvements, est proportionnelle au module de l'exponentielle. On pent écrire :

$$
2 h=2 h_{0}\left|e^{-i a x}\right| .
$$

A proximité du fond $(z=$ env. $-\mathrm{H})$, les valeurs $\operatorname{ch} \beta z$ et $\operatorname{sh} \beta z$ figurant dans l'expression de $\Psi^{*}$ et de la vitesse $u=\partial \Psi / \partial z$, sont pratiquement identiques et de signe contraire, mais leur module représente un nombre astronomique. Ceci est à l'origine de la variation extrêmement brusque des vitesses $u$, se comportant comme une véritable singularité (cf. fig. 2). Il y

sion paraissait probable a priori (notamment, on en a lait état dans le calcul approché fait au $\$ 1^{\mathrm{er}}$ ); encore ctait-il bon de s'en assurer.

Bien entendu, ce résultat n'infirme pas celui de M. Carry. Lorsque le fond est théoriquement lisse et les amplitudes assez faibles pour qu'on puisse admettre un mouvement entièrement non turbulent, il faut réintroduire la condition de vitesse nulle sur le fond. La valeur principale du taux d'amortissement est alors de l'ordre de $v^{1 / 2}$. Elle resterait telle si le fluide n'adhérait pas à la paroi, c'est-à-dire si la vitesse n'était pas nulle sur le fond, mais différerait nettement néanmoins de la valeur de hase (celle pour un fluide parfait). Même dans ce cas, on aboutirait, dès que les amplitudes sont sensible, à des gradients de vitesse très forts sur le fond, d'où la tendance à instabilité rendant la solution discutable. a lieu, par contre, de rechercher maintenant une solution d'allure régulière « sur le fond $»$. Le seul moyen d'y parvenir, quelque petit que soit $v$, est d'admettre les constantes C et D identiques; dans ces conditions:

$$
\mathrm{C} \operatorname{ch} \beta z+\mathrm{D} \operatorname{sh} \beta z=\mathrm{C} e^{\beta z}
$$

se transforme en une quantité extrêmement petite pour $z=-\mathrm{H}$.

Il suffit ensuite de se conformer aux calculs de MM. Bresel et Carry; on posera, pour simplifier :

$\mathrm{K}=\operatorname{ch} \alpha \mathrm{H}, \quad \mathrm{M}=\operatorname{sh} \alpha \mathrm{H}$,

$\mathrm{N}=\operatorname{ch} \beta \mathrm{H}, \quad \mathrm{P}=\operatorname{sh} \beta \mathrm{H}, \quad(\mathrm{N}-\mathrm{P})=\mathrm{S}=e^{-\beta \mathrm{H}}$

et obtiendra les trois équations suivantes :

Vitesse parallèle au fond, soit :

$w=-(\partial \Psi / \partial \mathrm{x})=0$,

pour $z=-\mathrm{H} ; \mathrm{AK}-\mathrm{BM}+\mathrm{CS}=0$,

Composante normale de la pression constante en surface :

$\mathrm{A}(g \alpha / i b)+\mathrm{B}\left(2 \alpha^{2} y+i b\right)$

$$
+\mathrm{C}[(g \alpha / i b)+2 \alpha \beta \nu]=0,
$$

Effort tangentiel nul en surface :

$$
2 \mathrm{~A} \alpha^{2} \nu+\mathrm{C}\left(2 \alpha^{2} \nu+i b\right)=0 .
$$

La quatrième condition imposée par M. CARRY, $u=(\partial \Psi / \partial z)=0$ sur le fond, est supprimée et remplacée par $\mathrm{C}=\mathrm{D}$.

Le déterminant des trois équations précédentes, linéaires et homogènes en $A, B, C$ doit être nul. En le développant, il vient :

$$
\begin{aligned}
\mathrm{K}\left(2 \alpha^{2} \nu+i b\right)^{2} & +\mathrm{M}\left(g \alpha-4 \alpha^{3} \beta \nu^{2}\right. \\
& -\mathrm{S}\left(2 \alpha^{2} \nu+i b\right) 2 \alpha^{2} \nu=0 .
\end{aligned}
$$

$S$ est extrêmement petit (son module serait de l'ordre de $10^{-22}$ pour l'exemple numérique indiqué au paragraphe I, $a$, pour lequel, cependant, $\mathrm{H}=2 \mathrm{~cm}$ est très faible) et peut être omis sans inconvénient. (33) s'écrira, en définitive :

$$
\left(2 \alpha^{2} y+i b\right)^{2}=-\alpha \operatorname{th} \alpha \mathrm{H} \times\left(g-4 \alpha^{3} \beta \nu^{2}\right.
$$

Cette équation fixe $\alpha$, car $\beta$ peut s'exprimer, en fonction de $\alpha$ et $\nu$, au moyen de la relation (29). On posera :

$$
\alpha=a+\mathrm{C}_{2} \nu^{1 / 2}+\mathrm{C}_{3} \nu+\mathrm{C}_{4} \nu^{3 / 2}+\ldots,
$$

les constantes $C_{n}$ étant obtenues, successivement, en égalant, dans les deux membres de (34), les termes de même puissance en v.

Le développement du facteur entre parenthèses du membre de droite de l'équation (34) fournit le terme constant $g$, le suivant étant d'ordre $\nu^{3 / 2}$ d'après la forme de $\beta ; \alpha$ th $\alpha \mathrm{H}$ comprend 
le terme constant $a$ th $a \mathrm{H}$, puis un terme non $n u l$ en $v^{1 / 2}$; multiplié en outre par $\mathrm{C}_{2}$; pour le membre de gauche, le développement débute par le terme constant $-b^{2}$, puis par un terme en $v$.

II s'ensuit immédiatement :

$$
\mathrm{C}_{2} \equiv 0
$$

* ne comporte pas de terme en $v^{1 / 2}$, par consćquent, selon (30), il en est de même pour la loi d'amortissement des amplitudes. Développons alors, en tenant compte de la condition (36), les deux premiers termes des facteurs apparaissant dans l'équation (34):

z th $\alpha \mathrm{H}=a$ th $a \mathrm{H}+\mathrm{C}_{3} \vee\left(1+\frac{2 a \mathrm{H}}{\operatorname{sh} 2 a \mathrm{H}}\right)$ th $a \mathrm{H}+\ldots$ $=a \operatorname{th} a \mathrm{H}+2 q \vee \mathrm{C}_{2}$ th $a \mathrm{H}+\ldots$

$$
\left(2 \alpha^{2} \nu+i b\right)^{2}=-b^{2}+4 a^{2} i b \nu+\ldots
$$

Il s'ensuit, puisque le facteur entre parenthèses du membre de droite de (34) ne fournit pas de terme en $v$ :

$$
b^{2}=g a \text { th } a \mathrm{H}
$$

relation valable, dans un fluide parfait, entre les paramètres $a$ et $b$, puis :

$$
\mathrm{C}_{3}=-\frac{2 i b a^{2}}{g q \operatorname{th} a \mathrm{H}}=-\cdot \frac{2 a^{2} i}{V^{\gamma} q}=-\frac{2 a^{2} i}{\mathrm{G}}
$$

D'après l'équation (30), l'amplitude décroîtra selon la loi exponentielle suivante :

$$
2 h=2 h_{0}\left|e^{-i \alpha x}\right|=2 h_{0} e^{-2 a^{2} \nu x / G},
$$

si l'on néglige les termes en $y^{3 / 2}$ et plus.

En dérivant, il viendra :

$$
\frac{\Delta \mathfrak{f}^{2} h}{\Delta x}=-\frac{2 a^{2} \nu}{\mathrm{G}}
$$

formule identique $\dot{a}$ (13), si l'on remplace $Q$ par $\mathrm{Q}_{1, \nu}$ [formule (15)], ce qui achève la démonstration de la propricté énoncée plus haut.

Il s'ensuit aussi que la valeur principale de l'énergie dissipée par la viscosité interne est donnée par la formule (15), proportionnelle au coef_. ficient cinématique $v$.

\section{RÉFÉRENCES BIBLIOGRAPHIQUES}

[1] BakhmetefF (B. A.). - Mécanique de l'écoulement turbulent des fluides. Paris, Dunod, 1941.

[2] BuÉser (F.). - Calcul de l'amortissement d'unc houle dans un fluide visqueux de profondeur finie. La Houille Blanche, 1949, pp. 630-634.

[3] Carry (C.). - Calcul de l'amortissement d'une houle dans un fluide visqueux en profondeur finie. la Houille Blanche, 1956 , pp. 75 et suivantes.

[4] Emmons (H. W.). - The laminar-turbulent transition in a boundary layer. Part I. Jour. Aero. Sci., vol. $18, \mathrm{n}^{\circ} 7$, p. 490 , July 1951.

[5] Huov Lx. - Stability of oscillatory laminar flow along a wall. Beach Erosion Board. Technical Memorandum $\mathrm{n}^{\circ} 47,1954$.

[6] Lamb (Sir H.). - Hydro-dynamics. Dover, New-York, 1945 (p. 622).
[7] Larras (J.). - Effet de la houle et du clapotis sur les fonds sableux. Paris, $I V^{o s}$ Journies de l'Itydraulique, 1956.

[8] Mrche (R.). - Mouvements ondulatoires de la mer. Annales des Ponts et Chaussées. Janvier it août 1944 .

[9] Miche (R.). - Propriétés des trains d'ondes océaniques et de laboratoire. Paris, Comité Central d'Océanographie et d'Etude des Cótes, 1954.

[10] Valembors (J.). - La mesure des pressions variables. Paris, Hermann et Cie, 1948 (p. 42).

[11] Valembois (J.). - Quelques considérations sur la similitude dans les essais de houle sur modèle. Paris, IVes Journées de l'Hydraulique, 1956.

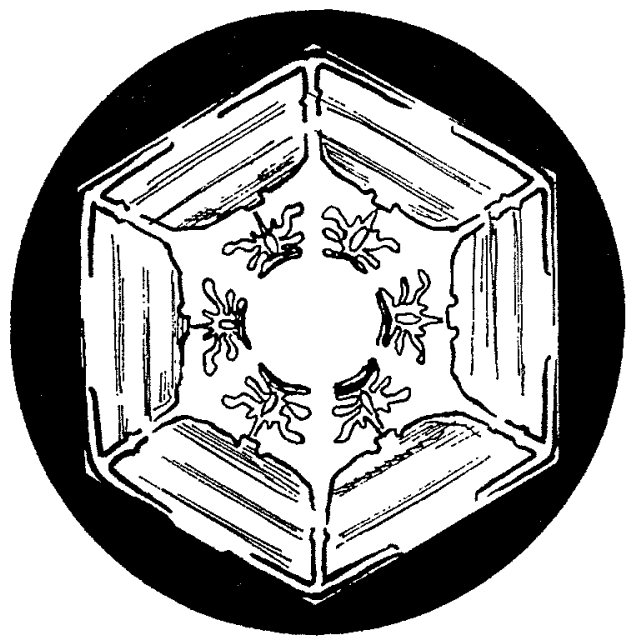

